Article

\title{
Disinhibited-like behavior correlates with frontal cortex damage in alcohol-induced Wernicke-Korsakoff Syndrome
}

\author{
Marta Moya 1,2, Leticia López-Valencia1, Borja García-Bueno³ and Laura Orio 1,2,*
}

1 Department of Psychobiology and Behavioral Sciences Methods, Faculty of Psychology, Universidad Complutense de Madrid (UCM), Madrid, Spain; martamoyamontes@ucm.es (M.M.) ; ; letlop01@ucm.es (LLV); lorio@psi.ucm.es (L.O.).

2 Red de Trastornos Adictivos (RTA) del Instituto de Salud Carlos III (ISCIII)

3 Departament of Pharmacology and Toxicology, Faculty of Medicine, UCM \& Instituto de Investigación Sanitaria Hospital 12 de Octubre (Imas12), Instituto Universitario de Investigación en Neuroquímica IUINUCM, Avda. Complutense s/n, 28040 Madrid, Spain. Centro de Investigación Biomédica en Red de Salud Mental, CIBERSAM (Biomedical Network Research Center of Mental Health), Institute of Health Carlos III, 28029 Madrid, Spain. bgbueno@med.ucm.es (BGB)

* Correspondence to: lorio@psi.ucm.es ; Department of Psychobiology and Behavioral Sciences Methods, Faculty of Psychology, Complutense University of Madrid, Campus de Somosaguas s/n. 28223 Pozuelo de Alarcón (Madrid), Spain

\begin{abstract}
Wernicke-Korsakoff syndrome (WKS) is induced by thiamine deficiency (TD) and mainly related to alcohol consumption. Frontal cortex dysfunction has been associated to impulsivity and disinhibition in WKS patients. The pathophysiology involves oxidative stress, excitotoxicity and inflammatory responses leading to neuronal death, but the relative contributions of each factor (alcohol and TD, isolate or in interaction) to these phenomena are still poorly understood. A rat model was used by forced consumption of $20 \%(\mathrm{w} / \mathrm{v})$ alcohol for 9 months (CA), TD hit (TD diet + pyrithiamine $0.25 \mathrm{mg} / \mathrm{kg}$, i.p. daily injections the last 12 days of experimentation; TDD), and both combined treatments (CA+TDD). Motor and cognitive performance and cortical damage were examined. CA caused hyperlocomotion as a possible sensitization of ethanol-induced excitatory effects and recognition memory deficits. In addition, CA+TDD animals showed a disinhibited-like behavior, which appears to be dependent on TDD. Also, combined treatment led to more pronounced alterations in nitrosative stress, lipid peroxidation, apoptosis and cell damage markers. Correlations between injury signals and disinhibition suggest that CA+TDD disrupts behaviors dependent on the frontal cortex. Our study sheds light on the potential disease-specific mechanisms, reinforcing the need for neuroprotective therapeutic approaches along with preventive treatments for the nutritional deficiency in WKS.
\end{abstract}

Keywords: Chronic alcohol 1, Thiamine Deficiency 2, Disinhibition 3, Wernicke's Encephalopathy 4, Recognition memory 5, Nitrosative stress 6, Lipid peroxidation 7, Apoptosis 8, Cell damage 9 Nutritional deficit 10

\section{Introduction}

Alcohol is one of the most widely used psychoactive drugs worldwide, whose consumption originates major public health problems in our society, including an alcohol use disorder (AUD) (reviewed in [1]). The confluence of nutritional deficiency in AUD patients is of particular interest, specifically in the case of thiamine deficiency (TD), as this can result in Wernicke-Korsakoff Syndrome (WKS). WKS is often characterized by ataxia, oculomotor disturbances, severe memory impairment, global confusion, executive dysfunction and apathy, mainly seen in chronic alcohol consumption cases. Ethanol affects 
thiamine bioavailability by multiple mechanisms such as reducing effective absorption, causing defective phosphorylation (thiamine diphosphate is the metabolically active form) and reducing thiamine storage in the liver because of an alcoholic liver disease [2]. Approximately $75 \%$ of TD cases are undiagnosed in AUD patients (reviewed in [3]). Other causes inducing the pathology, independent of alcohol, include chronic malnutrition caused by prolonged fasting and hyperemesis gravidarum, bariatric and gastrointestinal surgeries, acute pancreatitis, hemodialysis, etc. [4,5].

Thiamine is central to the preservation of the myelin sheath, neurotransmitter synthesis and cellular energy reserves. Impaired oxidative metabolism in TD due to decreased activity of thiamine-dependent enzymes leads to a multifactorial cascade of events in the brain that include focal decreases in energy status, decreased glucose utilization, lactic acidosis, blood-brain barrier disruption, astrocyte dysfunction, glutamatemediated excitotoxicity, amyloid deposition, oxidative stress and inflammation [6].

WKS patients have obvious cognitive and motor dysfunction, requiring heavy dependence to complete daily life activities. The most apparent neuropsychological deficits are memory alterations. However, these alterations are not the only ones, and on many occasions they are not the most limiting. The important alterations that they suffer in the field of affectivity and executive functions are much more devastating than memory problems. These patients frequently show affective flattening and lack the drive to start activities, which implies a loss of spontaneity and initiative $[7,8]$. Poor risk assessment and altered behavioral inhibition are also frequently encountered [9]. Disinhibition is a debilitating problem leading to impulsive and socially inappropriate behavior [10]. Thus, the set of neuropsychological characteristics indicated suggests a dysfunction of the frontal structures. There are several neuroimaging studies that indicate alterations in the frontal lobes of patients with WKS [11-14]. The marked apathy and lack of initiative observed in patients with WKS has also been found in many subjects with frontal injury [15].

Likewise, evidence indicates that the frontal cortex is important in behavioral inhibition, including cognitive processes, social behavior, and inhibition of motor responses. Damage to the frontal cortex lowers performance in executive control tasks, most likely by disrupting inhibition [10].

The frontal cortex is a structure vulnerable to damage by both alcohol and TD, as it has been described in previous studies (reviewed in [16]), including some of our research group [17,18].

In uncomplicated AUD patients and comorbid AUD patients with WKS, the prefrontal cortex is more vulnerable to chronic ethanol exposure than other cortical regions [19], as there are decreases in neuron density (15-23\%) [20], shrinkage of frontal lobe volume [21], and decreased metabolic rate (reviewed in [16]). Similar neuropathological changes in frontal cortex have been detected in non-alcoholic WKS patients [22].

Phenomena such as oxidative/nitrosative stress, lipid peroxidation, excitotoxicity and inflammation have been associated with cell damage and neurodegeneration induced by chronic alcohol and TD in this brain region (reviewed in $[1,16]$ ).

Thus, both ethanol toxicity and TD result in cortical damage and cognitive problems, but the relative contributions of each factor to these phenomena and the underlying mechanisms are still poorly understood.

Here, we examined the effects of chronic alcohol exposure (9 months), TD diet and the combination of both in neurotoxic parameters (nitrosative stress, lipid peroxidation, apoptosis and cell damage) in frontal cortex and in behavioral tasks that are highly dependent on this brain region, to further understand the independent effects of each treatment and how they interact.

\section{Materials and Methods}

\subsection{Animals and housing}

Male Wistar rats (Envigo@, Barcelona, Spain) $(n=50)$, weighing 100-125 g at arrival (postnatal day [PD] 28, approximately) were used. Animals were housed in groups of 23 per cage and maintained at a constant room temperature $\left(21 \pm 1{ }^{\circ} \mathrm{C}\right)$ and humidity $(60 \pm$ 
$10 \%$ ) in a reversed $12 \mathrm{~h}$ dark-light cycle (lights on at 8:00 p.m.). Standard food and tap water were available ad libitum during an acclimation period of 12 days prior to experimentation and then rats were randomly assigned to the experimental groups. Thus, animals began the experiments when they were around PD 40 (adolescent period). The number of animals in the alcohol and TD groups was slightly higher to control groups for the possible loss of experimental subjects.

All procedures followed ARRIVAL guidelines and were adhered to the guidelines of the Animal Welfare Committee of the Complutense University of Madrid (reference: PROEX 312-19) in compliance with the Spanish Royal Decree 53/2013 and following the European Directive 2010/63/EU on the protection of animals used for research and other scientific purposes.

\subsection{Experimental Groups}

In order to explore the different conditions that contribute to developing WKS, the following experimental groups were used:

- Chronic alcohol (CA)

A protocol based on Fernandez et al. (2016) [23] was employed. The ethanol solution was prepared from ethanol $96^{\circ}$ (Iberalcohol S.L., Madrid, Spain) in tap water, to the appropriate $\mathrm{v} / \mathrm{v}$. All rats had limited access to a single bottle, so the alcohol groups were exposed to forced consumption of an ethanol solution $(20 \%, \mathrm{w} / \mathrm{v})$ as the sole source of liquid. Alcohol was gradually introduced using a "fading on" procedure: ethanol exposure started at $6 \%$ for 5 days, followed by another 5 days at $9 \%, 5$ days at $12 \%, 2$ days at $16 \%$ and finally reached $20 \%$, maintained during the 36 weeks of duration of the experiment. CA rats $(n=9)$ were provided with standard food ad libitum throughout the treatment.

\section{- $\quad T D \operatorname{diet}(T D D)$}

Since the beginning of the study, rats of TDD group $(n=9)$ had access to a single bottle with tap water and were fed with the standard food, but the last 12 days of the experiment, the chow was replaced by a thiamine-deficient diet (residual thiamine level in diet $<0.5$ ppm; Teklad Custom Diet, Envigo, Madison,WI, USA), in conjunction with daily pyrithiamine hydrobromide (thiamine pyrophosphokinase inhibitor) injections (Sigma Aldrich, Madrid, Spain; 0.25 mg/kg; i.p.) (WKS Pre-Symptomatic Model (PSM) protocol in [18]).

\section{- Chronic alcohol combined with TD diet in the last days of treatment (CA +TDD)}

The rats received the same alcohol treatment as the CA group, and just like the TDD group, the last 12 days of the experiment, the standard food was replaced by the thiaminedeficient diet in conjunction with daily pyrithiamine hydrobromide injections $(0.25$ $\mathrm{mg} / \mathrm{kg}$; i.p.) $(n=10)$.

Furthermore, we included three control groups to monitor the alcohol and TD variables:

- Chronic alcohol with oral thiamine supplementation $(C A+T)$

We included a group receiving chronic alcohol with thiamine supplementation to deduce whether the effects of long term alcohol exposure can be ameliorated with prophylactic treatment $(n=8)$.

Since it is a chronic supplementation with thiamine, the route of administration chosen was oral, to avoid stress and discomfort of the animals produced by another type of procedure, such as daily injections for long periods of time. The pharmacological dose of thiamine hydrochloride (Sigma Aldrich, Madrid, Spain) chosen was of $0.2 \mathrm{~g} / \mathrm{L}$, taking as reference the ethanol exposure studies [24-28]. The drink containing $20 \%(\mathrm{w} / \mathrm{v})$ of alcohol was mixed with the thiamine, as the sole drinking fluid for the 36 weeks of the experiment. 
- $\quad$ Drinking water with oral thiamine supplementation $(C+T)$

Appropriate control subjects also received the same dose of $0.2 \mathrm{~g} / \mathrm{L}$ of thiamine in the water throughout the experiment $(n=6)$.

Since no significant changes were found in any of the variables analyzed, the data corresponding to the $C A+T$ and $C+T$ groups are not shown in the results, as will be explained later.

- Drinking water with standard chow (C)

Rats in the control group had access to a single bottle with tap water and standard chow ad libitum for the entire duration of the study $(n=8)$.

\subsubsection{Experimental procedures}

During the last 12 days of TDD protocol, the remaining animals $(C A, C A+T, C+T, C)$ received equivalent daily injections of saline (i.p.) to reproduce the same stress conditions in all animals.

Body weight of all animals was recorded weekly (every Monday, Wednesday and Friday), except during the final TDD protocol, where TDD and CA+TDD rats were weighed daily. Likewise, TD diet intake was also measured for these groups on those 12 days.

The volume of the bottles was measured and replenished every Monday, Wednesday and Friday. Additional bottles with the ethanol solution and tap water were included to control for spillage and evaporation during throughout the experiment. The grams of ethanol per kilogram of body weight was determined by the following formula: ([milliliters of ethanol consumed (loss control subtracted)] $x$ the percent concentration of ethanol $x$ the density of ethanol/ weight of the animal). Average daily ethanol consumption was determined by dividing the weekly consumption by seven days across the ethanol exposure phase.

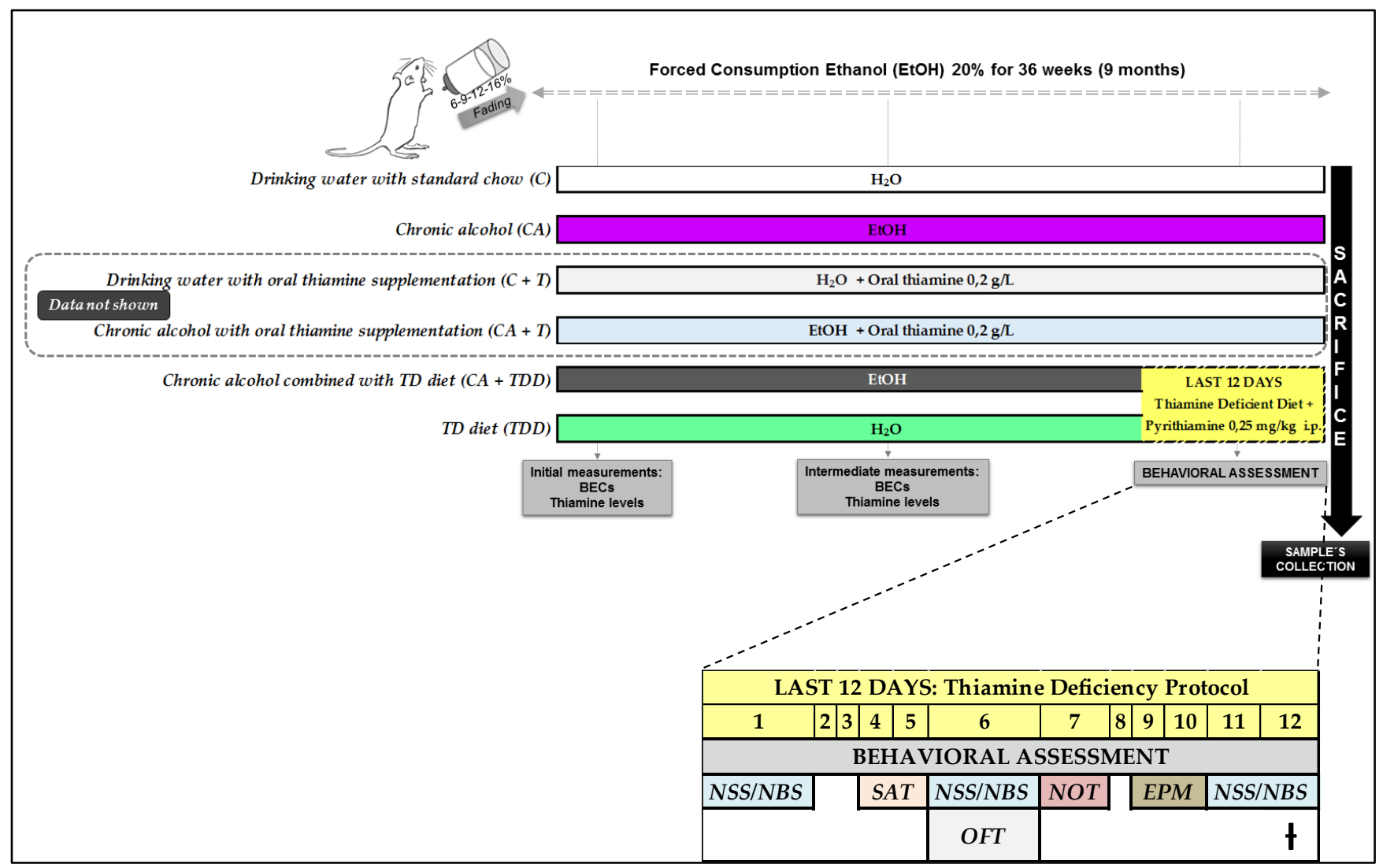


Figure 1. Experimental design: timeline of treatments and behavioral testing. Body weight and water $\left(\mathrm{H}_{2} \mathrm{O}\right) / \mathrm{ethanol}(\mathrm{EtOH}) \mathrm{con}-$ sumption were recorded throughout the entire experiment and the TD diet intake during the last 12 days. BECs: Blood Ethanol Concentrations; NSS/NBS: Neurological examination; SAT: Spontaneous Alternation Task; OFT: Open field Test; NOT: Novel Object Recognition Test; EPM: Elevated Plus Maze.

\subsection{Blood Ethanol Concentrations (BECs) and thiamine measurements}

During the chronic alcohol protocol, initial, intermediate and final measurements of BEC and thiamine were performed in all animals. Blood samples were collected from the tail in the weeks 4 and 12 of the experiment, and the final measurements were performed from trunk blood. Heparin $(5 \% \mathrm{w} / \mathrm{v})$ was used as anticoagulant, and plasma was obtained by blood centrifugation.

Ethanol levels were determined by using electrochemical detection of an enzymatic reaction with an AM1 Alcohol Analyzer (Analox Instruments, London, UK), and also by using the EnzyChrom ${ }^{\mathrm{TM}}$ Ethanol Assay Kit (BioAssay Systems, ECET-100) according to the manufacturer's protocol.

Thiamine was measured using an Enzyme-linked Immunosorbent Assay (ELISA) Kit for Vitamin B1 (VB1) (Cloud-Clone Corp.), following the manufacturer's instructions.

\subsection{Behavioral Assessment}

We focused on the evaluation of motor and cognitive domains, as we did in previous studies of WKS [18]. The time-course of behavioral assessments, appropriately scheduled not to interfere between them, is depicted in Figure 1. Behavioral tests were performed at least $1 \mathrm{~h}$ after any pharmacological treatment and were separated by a sufficient time interval to avoid interferences. Animals were evaluated following an alternation of experimental groups in all tests. All apparatus were carefully cleaned with ethanol $(15 \%, \mathrm{w} / \mathrm{v})$ between trials to remove possible odor cues. Analyses of behavioral test that were not computerized were performed by a double-blind protocol to ensure the reliability of the results.

- Neurological assessment

Motor and sensorial affectations, reflexes, walking and exploratory behavior in the home cage were evaluated by using the Neurological severity (NSS) and Neurobehavioral (NBS) scores, according to our previous studies [18]. This evaluation was carried out especially for the possible effects of the TD diet treatment. Therefore, all the animals were monitored on day 1,6 and 12 of the TDD protocol.

\section{- Motor function: locomotion}

The Open-field test (OFT) provides simultaneous measurement of locomotion and disinhibition-like behavior [29]. The apparatus is a square black box $(80 \mathrm{~cm} \times 80 \mathrm{~cm} \times 42 \mathrm{~cm})$ with the base divided into $6 \times 6 \mathrm{~cm}$ equal squares with legible white lines. Each rat was placed at the corner of the floor at the beginning of the trial, allowing the animal to freely move and explore the platform surface for $5 \mathrm{mins}$. Specific behavior parameters were registered using an automatic monitoring system (Smart software v. 2.0.14, Panlab). The general locomotor activity has been represented by the total distance travelled $(\mathrm{cm})$ and the walking speed $(\mathrm{cm} / \mathrm{s})$.

The Spontaneous Alternation Task (SAT) also evaluated locomotor activity. The test was carried out in a symmetrical Y-shaped maze, constructed of opaque black polyvinyl chloride (PVC). Each arm $(40 \times 15 \times 30 \mathrm{~cm})$ forms an angle of $120^{\circ}$ with the next, and they are equipped with visual signs on the walls. The testing room was dimly lit by a white lamp with luminosity between 15 and 17 lux. Each rat was allowed to freely explore the three arms of the maze for $8 \mathrm{~min}$ and the trials were video-recorded. The total number of arm entries was registered, considering an arm entry whenever a rat entered the arm with the four paws. 


\section{- Cognitive domain: disinhibition-like behavior and memory}

We also used the OFT to evaluate disinhibition. The open-field exposure paradigm is based on a conflict between the internal drive to explore a novel environment (based on the potential for rewarding outcomes) versus the internal drive to avoid a novel environment (based on the potential for aversive outcomes). Behavior in the OFT is influenced by various factors, including the level of illumination of the open-field arena. Bright light can be used as an aversive stimulus in the open-field paradigm leading to an increase in avoidance behaviors. Rodents are nocturnal and tend to avoid brightly lit places. Here, we performed the test under white light conditions (50-60 lux). In addition, rodents also avoid wide-open spaces and instead remain close to vertical references such as walls [30]. The platform surface was virtually divided into two regions, the center (inner zone) and the edges (in contact with the walls). Disinhibited rats show a lack of environmental awareness by spending more time in the arena center. The percentages of time spent and distance travelled in the inner zone compared to the total have been represented.

The Elevated Plus Maze test (EPM) is probably the most popular animal model of anxiety [31]. This model is based on a fear/curiosity balance towards novelty. When the typical rat behavior, with appropriate anxiety and general avoidance of open areas is altered, a disinhibited response could be observed [32,33]. The EPM is made of two black and grey plastic open arms $(50 \times 10 \mathrm{~cm})$ and two perpendicular closed arms of equal length and width but with $50 \mathrm{~cm}$ high opaque walls. A central area of $10 \mathrm{~cm}^{2}$ connected all arms. The platform was elevated $65 \mathrm{~cm}$ above the floor. When rats are placed in this test apparatus, they exhibit a strong preference for the closed-arms, indicating a fear reaction towards the open arms. This reaction is interpreted as accounting for anxiety [31]. Several factors may also influence the behavioural baseline (i.e., the open/total arm entries ratio), the ambient light intensity being amongst the most effective ones. Increasing the ambient illumination induces increased anxiety responses in the EPM. Here, we manipulated the intensity of anxiogenic stimuli by exposing them under white light of 55 lux conditions. The homogeneity of the light intensity was settled at the centre of the maze and at the extremity of the four arms by using a Luxmeter. The maximal difference measured between all parts of the maze never exceeded 5 lux. At the beginning of a test, each rat was placed on the central platform facing a closed arm and opposite to the experimenter position. Then, the animal was allowed to explore freely the maze for $5 \mathrm{~min}$. The number of entries and time spent in the open arms / the total arms (open and closed arms) were determined by a computer-controlled system recording the interruptions of infrared photo beams located along each arm. An entry in a given arm was counted when the rat had completely entered the arm (4 paws + tail in the arm). Data were analysed by using the MAZEsoft software (Panlab, Barcelona, Spain).

The $S A T$ also evaluated spatial working memory based on a natural tendency of rats to explore the less recently visited Y-maze arm [34]. Spontaneous alternation (successive entries into the three arms in overlapping triplet sets) index was calculated as the ratio of actual to possible alternations [(number of actual alternations/(total number of arm entries-2)] x 100] [35].

The Novel Object Recognition Test (NOT) analyzed possible impairments in the shortterm recognition memory. The task was performed in a square arena $(80 \times 80 \times 42 \mathrm{~cm})$ with black matte-painted walls and a bright black wooden floor and divided by white painted lines into 25 squares $(6 \times 6 \mathrm{~cm})$. The arena was subdivided into 4 equal sections, allowing the evaluation of 3-4 animals simultaneously. The NOR was carried out in accordance with previous studies [35] with some minor modifications. The test was performed in low light conditions (white light, 20 lux) and organized in three phases: Habituation (time=0), Training Phase (Pre-Test) and a Test Session $4 \mathrm{~h}$ after the Training Phase. During the habituation period, animals were allowed to freely explore the arena (without objects) for 5 min. On the Training Phase, two identical objects (glass bottles, the familiar objects (F) in 
the Test Session) were located in opposite corners of the arena and rats were allowed to freely explore them for $3 \mathrm{~min}$. In the Test Session, $4 \mathrm{~h}$ after the Training Phase, one of the familiar objects (F) was replaced by a novel object (N1, green ashtray), and animals were allowed to explore both objects for $5 \mathrm{~min}$. The objects with similar dimensions were selected, and their positions in the arena alternated in order to avoid possible place preferences. At the beginning of each session, the animals were placed in the corner of the apparatus facing the wall opposite to the objects. Both Training and Test Sessions were video recorded (Sony DCRDVD310E, Spain). Exploration of an object was considered whenever animals pointed their nose toward an object at a distance $\leq 1 \mathrm{~cm}$, while turning around, climbing and/or biting the objects was not considered as exploration. The time that animals spent exploring the objects during the test sessions, as well as the latency to first explore the novel object were registered. The discrimination index (DI) was calculated as the difference between the time spent exploring the novel object $(\mathrm{N})$ and the familiar one (F1 or F2) in relation to the total time spent exploring the objects $[(\mathrm{N}-\mathrm{F}) /(\mathrm{N}+\mathrm{F})]$. Negative values of the discrimination index were considered null.

\subsection{Blood and tissue samples collection}

The day 12 of TDD protocol, at least $1 \mathrm{~h}$ after treatment administration, animals were decapitated after lethal injection of sodium pentobarbital $\left(320 \mathrm{mg} / \mathrm{kg}\right.$, i.p., Dolethal ${ }^{\circledR}$, Vétoquinol, Spain). Blood for plasma determinations was collected from the trunk with tubes containing heparin ( $5 \% \mathrm{w} / \mathrm{v}, 1 \mathrm{vol}$ heparin per $9 \mathrm{vol} \mathrm{blood})$ as anticoagulant. Plasma was obtained from blood samples by centrifuging at $1000 \mathrm{~g}$ for $15 \mathrm{~min}$ at $4^{\circ} \mathrm{C}$. All plasma samples were stored at $-80^{\circ} \mathrm{C}$ until assayed. Brains were immediately isolated from the skull, discarding meninges and blood vessels, and the frontal cortex (area between Bregma +4.7 and $+1.2 \mathrm{~mm}$ aprox.) excised and frozen at $-80^{\circ} \mathrm{C}$ until assayed.

\subsection{Protein assay}

Protein levels were measured by Bradford's method (Bradford 1976) based on the principle of protein-dye binding.

\subsection{Western Blot Analysis}

Frontal cortex samples were homogenized by sonication in PBS $(\mathrm{pH}=7.4)$ mixed with a protease inhibitor cocktail (Complete, Roche ${ }^{\circledR}$, Madrid, Spain) in a dilution 1:3 (w/v), followed by centrifugation at $13000 \mathrm{rpm}$ for $10 \mathrm{~min}$ at $4^{\circ} \mathrm{C}$. After adjusting protein levels, homogenates were mixed with Laemmli simple buffer (Bio-Rad®, Alcobendas, Madrid, Spain) containing $\beta$-mercaptoethanol ( $50 \mu \mathrm{L} / \mathrm{mL}$ of Laemmli), to get a final concentration of $1 \mathrm{mg} / \mathrm{mL}$, and 15-20 uL was loaded into an electrophoresis gel. Proteins were blotted onto a nitrocellulose membrane (Amersham Iberica ${ }^{\circledR}$, Madrid, Spain) with a semidry transfer system (Bio-Rad), incubated with specific primary and secondary antibodies (see Table 1) and revealed by using an ECL ${ }^{\mathrm{TM}}-\mathrm{kit}$ (Amersham Iberica, Madrid, Spain). Autoradiographs were quantified by densitometry (NIH ImageJ ${ }^{\circledR}$ software, National Biosciences, Lincoln, Nebraska USA) and expressed as optical density (O.D.). In all Western blot analyses, the housekeeping $\beta$-actin protein was used as loading control.

Each blot contained different biological replicates per group and three blots were run in separate assays. Results represent the average of the 3 technical replicates. Blot images above the results graphs were always taken from the same gel. Samples from all experimental groups were always loaded randomly. For this reason and in order to follow the same order of group presentation in the graphs along the manuscript (and excluding groups reported only in supporting information), some bands were spliced in the figures. Original images of uncropped blots can be visualized in the mandatory Supporting Information files. 
Table 1. Specific antibodies used in Western blotting to detect proteins of interest.

\begin{tabular}{|c|c|c|}
\hline Protein & Primary antibody ${ }^{1}$ & Secondary antibody \\
\hline \multicolumn{3}{|c|}{ Lipid peroxidation marker } \\
\hline \multirow[t]{2}{*}{ 4-HNE } & 1:1000 BSA $3 \%$ & Mouse (1:2000) \\
\hline & MAB3249 RED Systems & \\
\hline \multicolumn{3}{|l|}{ Cell damage markers } \\
\hline HSP70 & $1: 1000 s c-1060$ & Goat (1:4000) \\
\hline HSP60 & $1: 1000$ sc-13966 & Rabbit (1:2000) \\
\hline HMGB1 & $1: 1000 s c-56698$ & Mouse (1:2000) \\
\hline \multicolumn{3}{|l|}{ Apoptosis markers } \\
\hline Caspase 3 & $1: 500 s c-56053$ & Mouse (1:2000) \\
\hline Caspase 8 & $1: 400 s c-81656$ & Mouse (1:2000) \\
\hline Caspase and pro-9 & 1:500 BSA 1\% sc-56076 & Mouse $(1: 2000)$ \\
\hline \multicolumn{3}{|c|}{ Nitrosative stress marker } \\
\hline iNOS & 1:500 BSA $2 \% s c-650$ & Rabbit (1:2000) \\
\hline \multicolumn{3}{|l|}{ Housekeeping } \\
\hline$\beta$-actin & 1:10000 A5441 Sigma & Mouse (1:10000) \\
\hline
\end{tabular}

${ }^{1}$ Dilutions and references (codes). Abbreviations: [4-HNE: 4-Hydroxynonenal; HSP70/HSP60: heat shock protein 70/60; HMGB1: high mobility group box 1 protein; iNOS: inducible oxide nitric synthase; BSA: Bovine serum albumin; sc: Santa Cruz Biotechnology]. Sources of secondary antibodies: Anti-Mouse IgG, HRP-linked whole Ab (from sheep) (GE Healthcare, ref. NXA931); Anti-Goat IgG (whole molecule)-Peroxidase antibody produced in rabbit (Sigma-Aldrich, ref. A5420); Anti-Rabbit IgG (H+L) Cross-Adsorbed Secondary Antibody, HRP conjugate (from donkey) (ThermoFisher Scientific, ref. 31458).

\subsection{Plasma nitrites $\left(\mathrm{NO}_{2}{ }^{-}\right)$levels}

As the stable metabolites of the free radical nitric oxide (NO·), $\mathrm{NO}_{2}{ }^{-}$were measured by using the Griess method [36]. In an acidic solution with $1 \%$ sulphanilamide and $0.1 \%$ $\mathrm{N}$-(1-Naphthyl) ethylenediamine (NEDA), nitrites convert into a pink compound that is photometrically calculated at $540 \mathrm{~nm}$ in a microplate reader. The results were expressed as percentage of control.

\subsection{Statistical Analysis}

Data, expressed as mean \pm S.E.M, were analyzed by two-way ANOVA (with repeated measures for weight and consumption data) when normality was verified, otherwise by Kruskal-Wallis test. Homocedasticity was checked by Barlett's test and data transformed (sqrt) when appropriate. In the ANOVA, Bonferroni post hoc test were used when appropriate. Outliers were analyzed using the Grubbs' test. Correlations between behavioral and biological measures were assessed by Pearson's and linear regression analyses. To account for multiple testing in the correlations, we adjusted the p-values by controlling the false discovery rate (FDR), which was set to 0.05 and assessed with the BenjaminiHochberg procedure. This type of correction allows restricting the occurrence of falsepositive findings (type I error) among all nominal significant findings. A p value $<0.05$ was set as the threshold for statistical significance in all statistical analyses. The data were analyzed using GraphPad Prism version 8.0 (GraphPad Software, Inc., La Jolla, CA, USA).

\section{Results}

\subsection{Weight and consumptions}

3.1.1. Body weight throughout the 36 weeks of experiment and TD diet intake

All rats gained weight during the course of the experiment (Figure 2A; two-way repeated measures (RM) ANOVA; interaction between factors (time $\times$ treatment), $\mathrm{F}(2,68)=$ 7.006, $p=0.0017$; and main effects of time: $F_{(1,122,38,15)}=1360, p<0.0001$ and treatment: $F_{(1,34)}$ 
$=27.43, \mathrm{p}<0.0001$; within-group differences, $\mathrm{p}<0.0001)$, but $\mathrm{CA}$ animals weighed significantly less than control subjects during all weeks (between-group differences, CA vs $\mathrm{C}$ weeks 1-12 and 13-24: $\mathrm{p}<0.0001$; weeks 25-34: $\mathrm{p}=0.0002$ ).

With the start of TD diet, the four corresponding groups were fully shaped and a significant weight loss was observed in TDD and CA+TDD animals at the end of the protocol (Figure 2A, right box up; two-way RM ANOVA, interaction: $\mathrm{F}(3,32)=4.742, \mathrm{p}=0.0076$; effect of time: $F_{(1,32)}=30.24$, $p<0.0001$; effect of treatment: $F_{(3,32)}=8.26, p=0.0003$; both groups: $\mathrm{p}=0.0002$ ).

This weight loss in TDD and CA+TDD groups is consistent with a decrease in their food intake (Figure 2A, right box down; two-way RM ANOVA, interaction: $\mathrm{F}(1,17)=8.932$, $\mathrm{p}=0.0083$; effect of time: $\mathrm{F}(1,17)=745,8, \mathrm{p}<0.0001$; both groups: $\mathrm{p}<0.0001)$.

\subsubsection{Alcohol intake and BECs}

As shown in Figure 2B, there were differences in the amount of alcohol consumed across treatment (RM one-way ANOVA, $\mathrm{F}(1,259,22,67)=15.54, \mathrm{p}=0.0003)$. In the early adolescent stages and being younger, the rats drank more than during the adult stages (weeks 13-24 vs 1-12: $\mathrm{p}<0.0001$; weeks 25-34 vs 1-12: $\mathrm{p}=0.0001$ ).

During TDD treatment, the CA+TDD rats displayed an increase in ethanol consumption between end and beginning of TD diet treatment (week 36 versus 35) (two-way RM ANOVA, overall effect of time, $F(1,17)=5.859, p=0.027 ; p=0.0478$ ) which was not observed in the CA group ( $p>0,05$, n.s.). No significant differences were found between CA groups with and without TD diet ( $p>0.05, n . s)$.

Blood ethanol levels (indicated in Figure 2B, boxed numbers), measured at 1 month, 3 months and 9 months of CA (weeks 4,12 and 36), did not change significantly across months $(\mathrm{F}(2,17)=0.4223, \mathrm{p}>0.05, \mathrm{n} . \mathrm{s})$. The global BEC mean was of $73.33 \pm 12.65 \mathrm{mg} / \mathrm{dL}$, modeling moderate drinking. Samples from some randomized control animals were also measured as technical controls, obtaining values ranging from 3 to $7 \mathrm{mg} / \mathrm{dL}$ 


\section{A. Body weight and TD food intake}

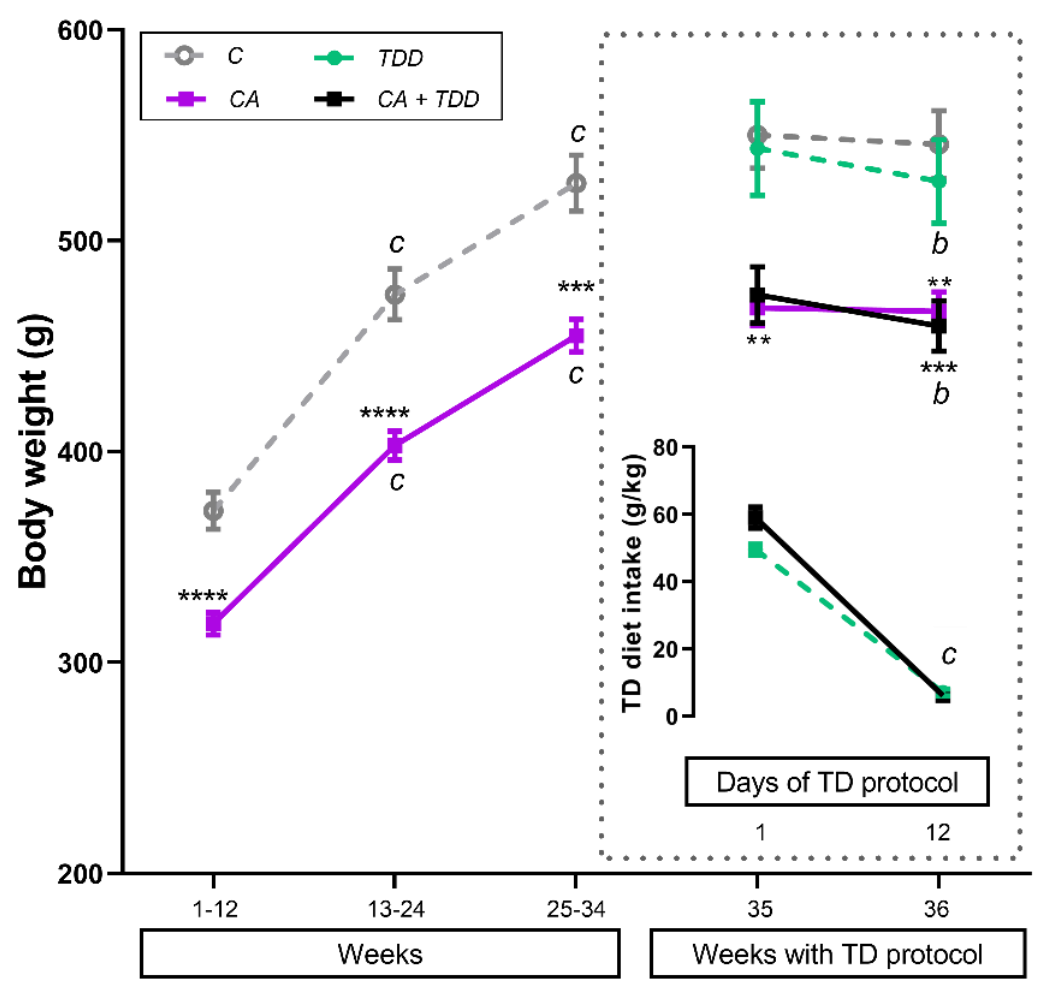

\section{B. Alcohol consumption}

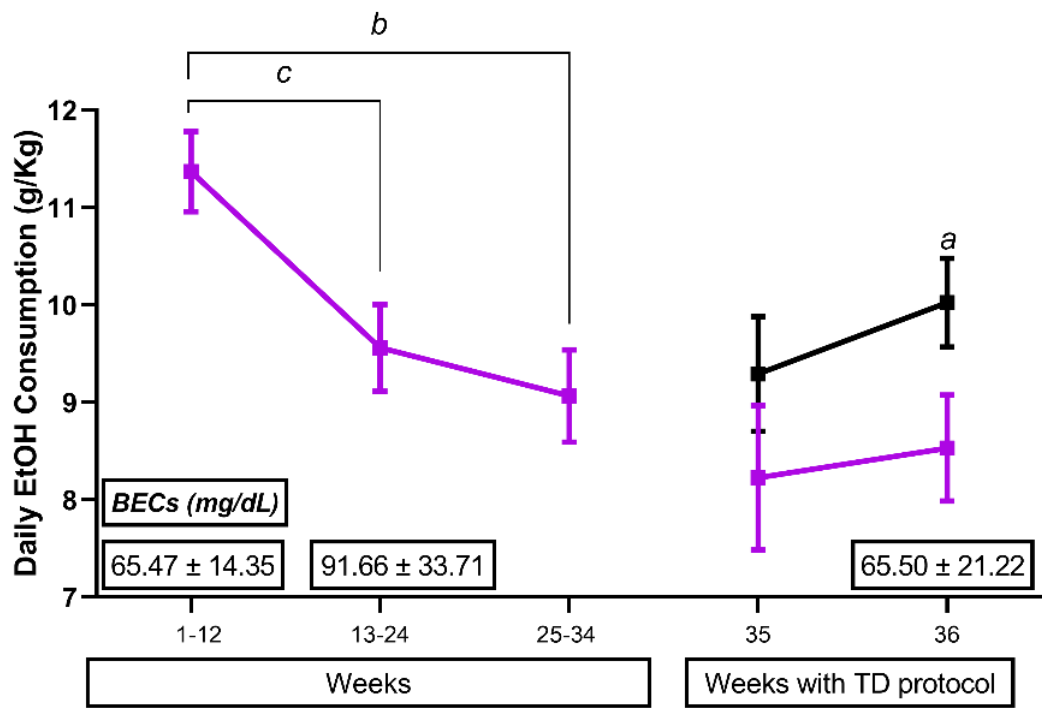

Figure 2. Physiological parameters along the procedure. (A) Average weights at weekly intervals and at days 1 and 12 of the final TD diet protocol, along with the diet intake $(\mathrm{g} / \mathrm{Kg})$. All rats gained weight until week 34 , but the CA animals were always below the controls. Weight loss in TDD and CA+TDD groups during TD diet treatment was consistent with the decreased intake. (B) Ethanol consumption $(\mathrm{g} / \mathrm{Kg}$ ) across weeks, along with Blood Ethanol Concentrations (BECs (boxed numbers): mean \pm SEM). The animals consumed more alcohol during their adolescent and young stages than in the adult stage. All data are expressed as mean \pm S.E.M. Repeated measures ANOVAs; within-group differences: $a \mathrm{p}<0.05 ; b \mathrm{p}<0.001 ; c \mathrm{p}<0.0001$; between-group: different vs control group: $\left.{ }^{* *} \mathrm{p}<0.01,{ }^{* * *} \mathrm{p}<0.001 ;{ }^{* * *} \mathrm{p}<0.0001\right)$. 


\subsection{Neurological examination}

No significant neurological signs or behavioral disturbances were found in animals through NSS/NBS examination on days 1, 6 and 12 of the TDD protocol.

We explored all rats in the open surface and in their home cages, checking general activity, flexion of limbs and extension of head, movements of the tail, posture, reflexes, etc. Only some slight deficit in the startle reflex in a few alcohol-treated animals (CA and CA+TDD) was found, which seemed not to react to the sound stimulus (clap/finger snaps) as controls. Beyond this, no other symptoms or evidence of motor alteration, such as ataxia, was detected due to treatments effect.

\subsection{Behavioral outcomes}

3.3.1. Effects on locomotor activity and spatial memory: $S A T$

Two-way ANOVA revealed a main stimulatory effect of chronic alcohol on locomotor activity, since the number of total arm entries of the CA and CA+TDD rats was significantly increased (Figure 3.A, left graph, overall effect of alcohol $F(1,31)=5.152, p=0.0303$ ).

No effects in spontaneous alternation were observed due to alcohol or TDD, nor a significant interaction between these factors. Thus, spatial working memory did not appear to be affected by the treatments (Figure 3.A, right graph, $p>0.05$, n.s.)

\subsubsection{Effects on locomotor activity and disinhibition-like behavior: OFT}

As well as in the SAT, a significant overall effect of alcohol was found on OFT locomotor activity, increasing the total distance traveled and walking speed in the CA and $\mathrm{CA}+\mathrm{TDD}$ animals (Figure 3.B, upper left graph, $\mathrm{F}_{(1,32)}=6.859, \mathrm{p}=0.0134$; upper right graph, $\mathrm{F}(1,32)=7.348, \mathrm{p}=0.0107$; respectively).

In addition, there were an interaction between CA $\times$ TDD in the percentage of the distance travelled and time spent in the internal zone of the field (Figure 3.B, lower left graph, $\mathrm{F}(1,31)=4.246, \mathrm{p}=0.0478$; lower right graph, $\mathrm{F}(1,31)=5.444, \mathrm{p}=0.0263$; respectively). Post-hoc comparisons revealed a trend for group CA+TDD to walk more in the inner zone $(\mathrm{p}=0.066)$ and a significant effect of time spent in the inner zone respect to CA rats; $\mathrm{p}=$ $0.028)$. In this way, the rats treated by alcohol combined with TDD showed the highest percentages among all groups.

Thus, CA+TDD rats appeared to be disinhibited by preferring the internal area of the arena, which is known to be a more anxiogenic zone that the rest of the arena.

\subsubsection{Effects on disinhibition-like behavior: $E P M$}

As shown in Figure 3.C, animals exposed to CA along with TDD displayed a higher percentage of time spent in the open arms compared to controls $(p=0.0036)$, as well as versus CA $(\mathrm{p}=0.0039)$, the comparison with the TDD group was very close to the significance $(\mathrm{p}=0.0569)$ (left graph, Kruskal-Wallis=15.74, $\mathrm{p}=0.0013$ ).

Likewise, CA+TDD rats also explored the open arms significantly more than control $(\mathrm{p}=0.0151)$ and CA $(\mathrm{p}=0.0441)$ animals, indicated in percentage of entries (right graph, Kruskal-Wallis=11, $\mathrm{p}=0.0117)$.

Accordingly, similar to what was observed in the OFT, exclusively the combination of both treatments (CA+TDD) had a significant effect on disinhibition.

\subsubsection{Effects on recognition memory: NOT}

No differences were found in the total time that animals spent exploring the objects during the training session $(C=43.07 \pm 4.1 ; \mathrm{TD}=43.29 \pm 6.01 ; \mathrm{CA}=45.77 \pm 5.03 ; \mathrm{CA}+\mathrm{TD}=45.73$ $\pm 3.3 ; \mathrm{p}>0.05, \mathrm{n} . \mathrm{s})$.

The time of first approach to the novel object rendered a general significant effect of alcohol, so CA and CA+TDD animals displayed higher latency (Figure 3.D, left graph, F $(1,30)=7.652, \mathrm{p}=0.0096)$.

Furthermore, the two-way ANOVA of the discrimination index data also demonstrated a significant alcohol effect, decreasing their recognition capacity (Figure 3.D, right graph, $\mathrm{F}(1,29)=4.557, \mathrm{p}=0.0414)$. 
Therefore, chronic exposure to alcohol seemed to cause a cognitive impairment by worsening the memory capabilities of CA and CA+TDD animals.

A. Spontaneous Alternation Task (SAT)

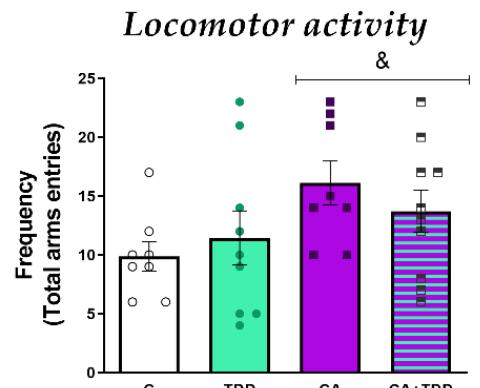

Spatial memory
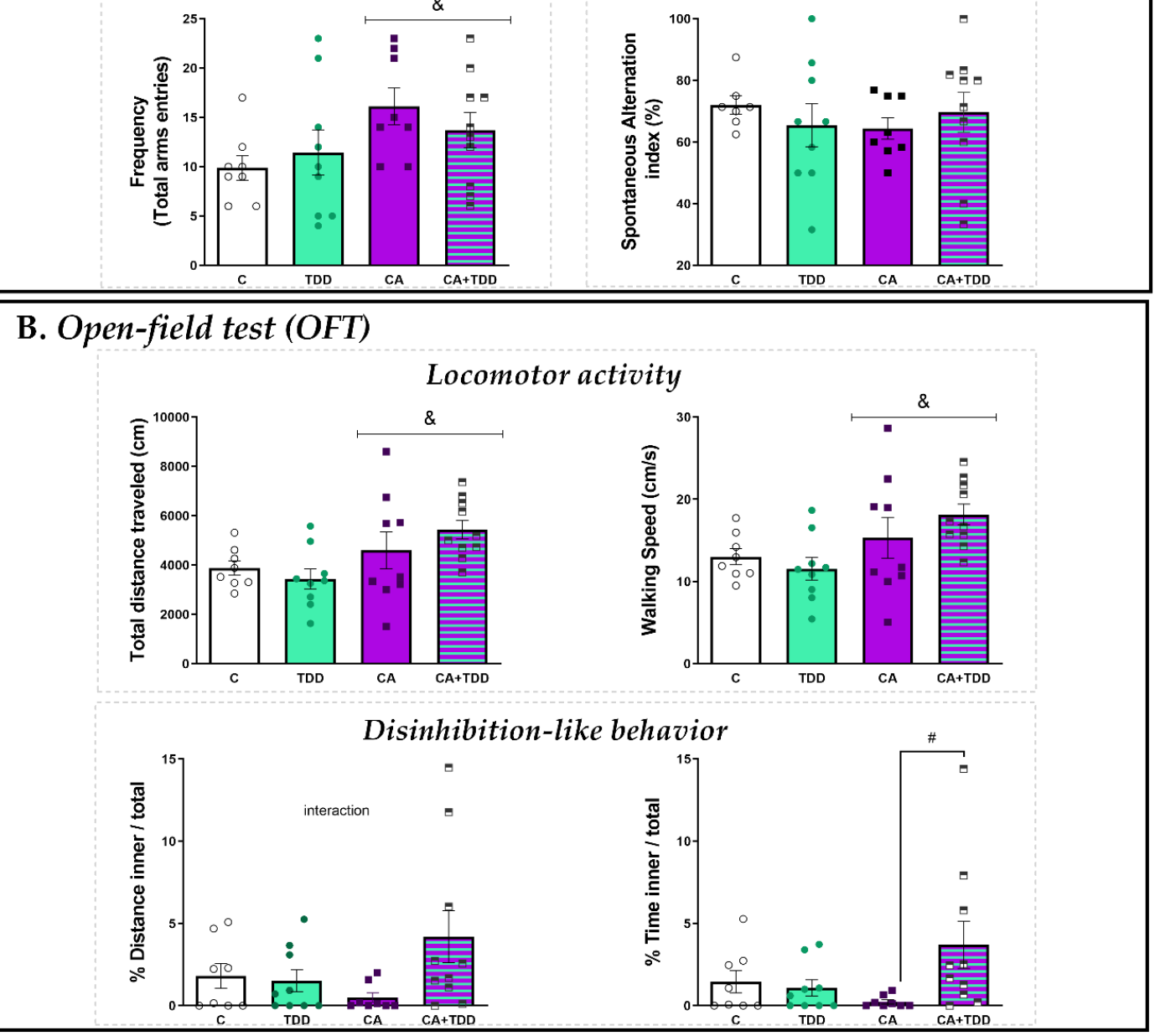

C. Elevated Plus Maze test (EPM): disinhibition-like behavior
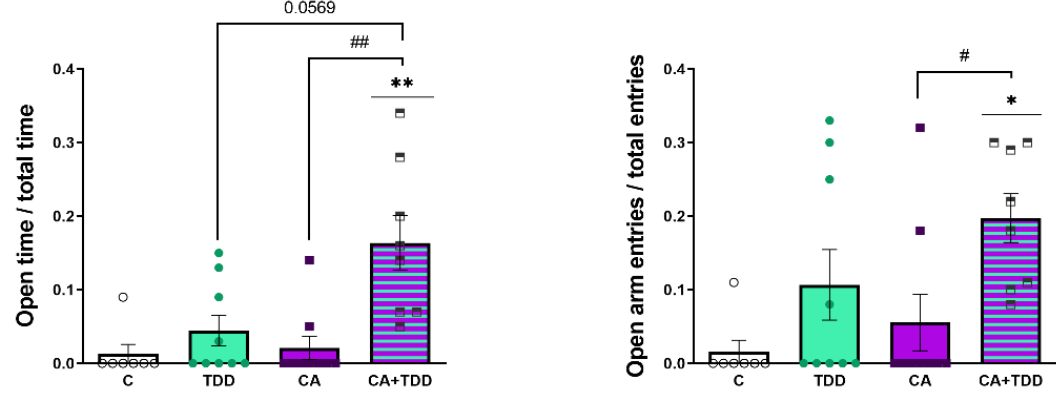

D. Novel Object Recognition Test (NOT): recognition memory
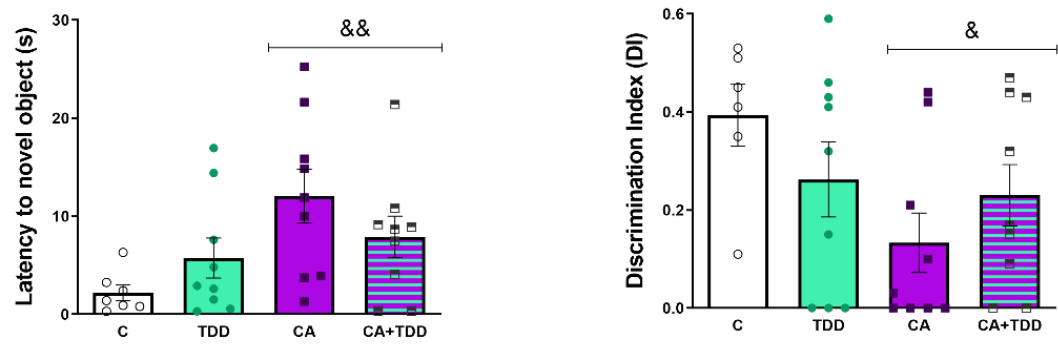
Figure 3. Behavioral changes as a function of treatment condition. (A) Spontaneous alternation task (SAT). There was an increase of the total arm entries by CA groups, independently of TDD treatment, interpreted as hiperlocomotion, and no effect on spontaneous alternation reflecting spatial working memory. (B) Open field (OFT) testing locomotion and disinhibition. Here, we also found motor agitation by alcohol. Rats typically avoid open areas. CA+TDD-treated rats explored more time the inner zone than the rest of group, reflecting a trend of disinhibition. (C) Elevated plus maze (EPM). Time spent and entries in open arms showed a remarkable disinhibited behavior just in rats with CA combinated with TDD treatment. (D) Effect on memory abilities tested by NOT. Rats exposed to CA were impaired on the ability to discriminate the new object, thus showing a deficit in recognition memory. Mean \pm SEM $(n=8-10)$. Two-way ANOVA or nonparametric Kruskal-Wallis test. Overall alcohol effect: \&p $<0.05$, \&\&p $<0.01$; Interaction (CA x TDD) followed by post hoc test: different from control group: ${ }^{*} \mathrm{p}<0.05,{ }^{* *} \mathrm{p}<0.01$; different from CA: $\# \mathrm{p}<0.05$, \#\# $<0.01$.

\subsection{Alterations found in the frontal cortex and in plasma}

3.4.1. Effects on oxidative/nitrosative stress and lipid peroxidation markers

We measured markers that lead to nitrosative stress, such as the inducible nitric oxide synthase (iNOS) enzyme and the accumulation of the stable metabolite of $\mathrm{NO}, \mathrm{NO}^{-}$.

The protein levels of iNOS enzyme were analyzed in the frontal cortex, and no changes were detected (Figure 4.A, $\mathrm{p}>0.05$ n.s.). Nonetheless, the nitrites $\left(\mathrm{NO}^{-}{ }_{2}\right)$ levels were studied at the peripheral level (Figure 4.A). The analysis in plasma showed main effects of alcohol $(\mathrm{F}(1,31)=7.377, \mathrm{p}=0.0107)$ and TDD treatment $(\mathrm{F}(1,31)=5.924, \mathrm{p}=0.0209)$. Post hoc comparisons revealed that nitrites levels were significantly higher in the CA+TDD group compared to controls ( $\mathrm{p}=0.0073)$.

These results indicate that CA consumption interacts with TDD inducing a notable increase in markers of peripheral nitrosative stress.

Lipid peroxidation is a consequence of excessive oxidative/nitrosative cellular stress. We measured 4-hydroxynonenal (4-HNE) as an aldehydic product of lipid peroxidation. As shown in Figure 4.A, rats exposed to CA showed a significant increase in the 4-HNE protein levels compared to controls ( $\mathrm{p}=0.0006)$, this effect being even more pronounced by the combined treatment with TDD $(\mathrm{p}<0.0001)$ (Kruskal-Wallis=22.72, $\mathrm{p}<0.0001)$.

Thus, our results show that CA and CA+TDD treatments produce damage to the cellular lipid membranes in the frontal cortex cells.

\subsubsection{Effects on apoptotic markers}

Different caspases, such as 3, 8 and 9 could generate apoptotic cell death.

Respecting Caspase 3 and Caspase 8, there were significant differences between groups (Figure 4.B, Kruskal-Wallis $=9.062 ; 8.04, p=0.0285 ; 0.0452$; respectively). In particular, the group with the combination of CA and TDD treatments showed a trend to the highest levels of expression of these caspases, with levels very close to the significance for caspase $8(\mathrm{p}=0.0569)$ compared to control group.

In relation to Caspase 9, an interaction between factors was found $(F(1,32)=32.45$, $\mathrm{p}<0.0001)$, together with main effects of alcohol $(\mathrm{F}(1,32)=31.49, \mathrm{p}<0.0001)$ and TDD treatment $\left(\mathrm{F}_{(1,32)}=13.09, \mathrm{p}=0.001\right)$. Post hoc analysis revealed higher levels in all treated groups compared with the control group (Figure 4.B, for all comparisons, $\mathrm{p}<0.0001$ ).

\subsubsection{Effects on cell damage markers}

Some molecules can be released in response to injured tissue, such as heat shock proteins (HSPs) and the high mobility group box 1 protein (HMGB1).

We found significant changes in the HSP70 levels (Figure 4.C, Kruskal-Wallis=8.874, $\mathrm{p}=0.031$ ) only in the CA+TDD group, which were significantly higher than those in the control group ( $p=0.0325)$. There were not differences in the HSP60 expression levels between the different experimental groups (Figure 4.C, $p>0.05$ n.s.).

Regarding HMGB1 protein expression, there was a main effect (upregulation) of the TDD (Figure 4.C, $\mathrm{F}(1,30)=5.905, \mathrm{p}=0.0213$ ). Post hoc analysis also showed that the increase was greater in the CA+TDD group relative to controls $(\mathrm{p}=0.0377)$. 
(A) Nitrosative stress and lipid peroxidation
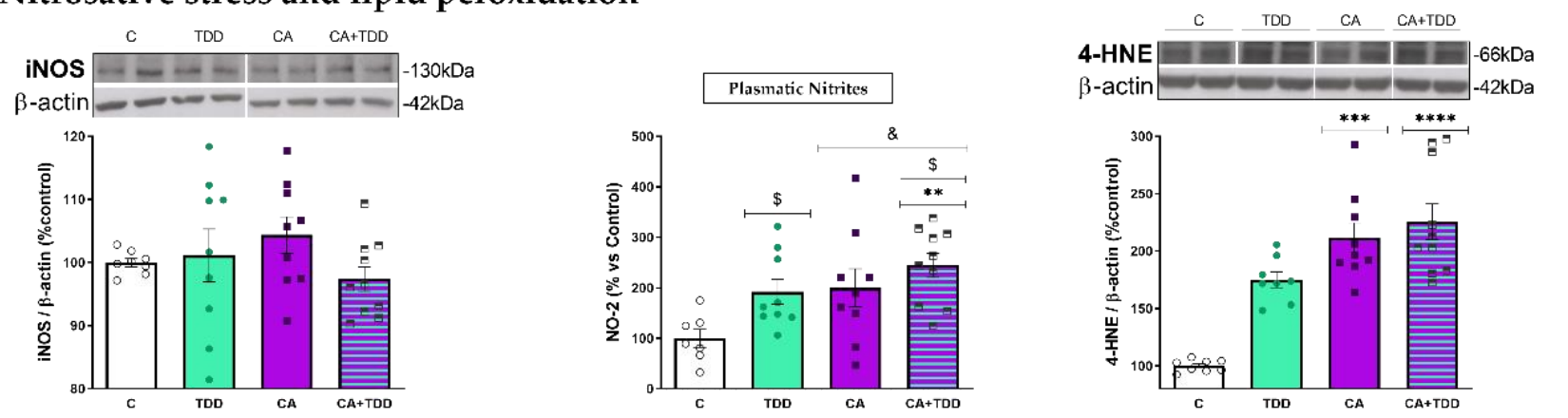

(B) Apoptotic cell death
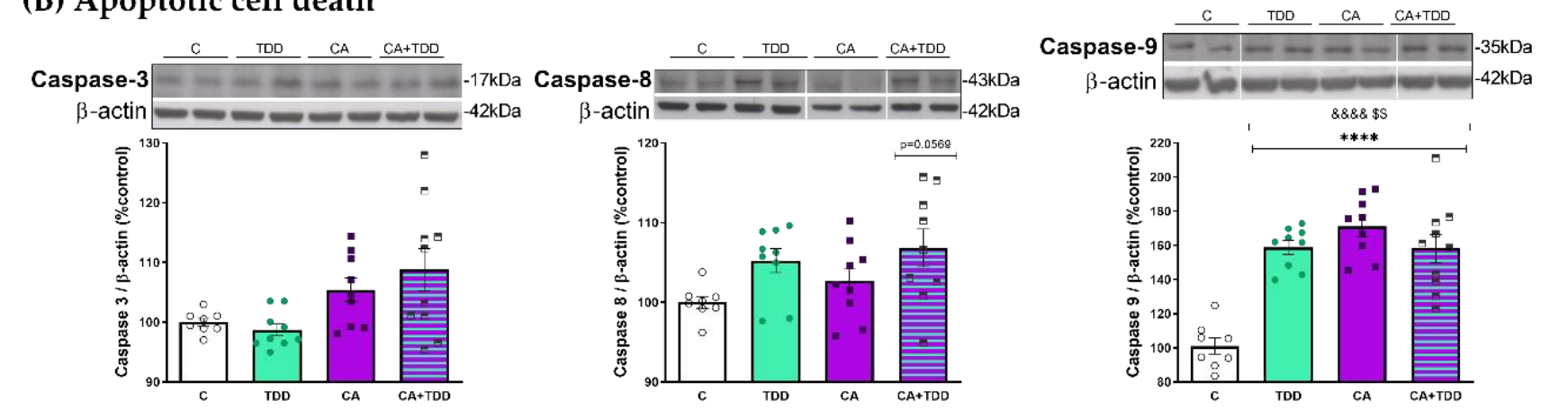

(C) Cell damage
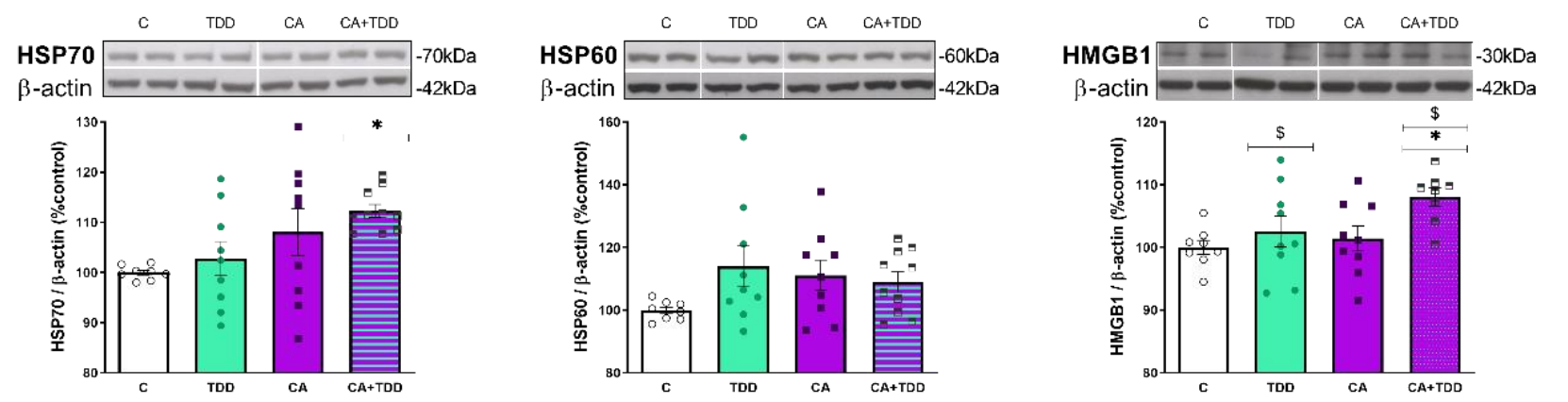

Figure 4. Biochemical changes as a function of treatment condition. Graphs indicate protein levels of markers in the frontal cortex and plasmatic nitrites. Western blot data of the respective bands of interest (upper bands) were normalized by $\beta$-actin (lower band) and expressed as a percentage of change versus the control group. (A) Nitrosative stress markers. Nitrites (NO-2) increased in plasma for all conditions exposure, but the combination of CA and TDD enhanced this elevation. Lipid peroxidation was measured by 4-Hydroxynonenal (4-HNE) accumulation. There was a significant effect of CA and CA+TDD groups versus control group. (B) Markers of apoptotic cell death: Caspase 3, 8 and 9. All treatments lead to an elevation of Caspase 9, relative to controls. (C) Damage-associated molecular patterns (DAMPS): HSP70, HSP60 and HMGB1. CA+TDD treatment increased the HSP70 and HMGB1 expression respect to controls. Mean \pm SEM $(\mathrm{n}=8-10)$. Two-way ANOVA or nonparametric Kruskal-Wallis test. Overall alcohol effect: $\& p<0.05, \& \& \& \& p<0.0001$; Overall TDD effect: $\$ p<0.05, \$ \$ p<0.01$; Interaction $(C A$ $\mathrm{x}$ TDD) followed by post hoc test: different from control group: ${ }^{*} \mathrm{p}<0.05,{ }^{* *} \mathrm{p}<0.01,{ }^{* * *} \mathrm{p}<0.001,{ }^{* * * *} \mathrm{p}<0.0001$.

\subsection{Correlations and linear regression analyses between biochemical and behavioral pa- rameters}

Here, we hypothesized that the signals of damage found in the frontal cortex of CA+TDD animals were, to some extent, related to the behavioral alterations observed. Exclusively in this model with the combination of both treatments, we found disinhibition 
as the clearest alteration in behavior. This dyfunction may reflect a deficit in executive functioning that has been related to frontal brain damage in WKS patients [9].

Then, considering the most noteworthy biochemical changes and behavioral outcomes in our combinated model, we performed correlation analyses that are summarized in Table 2. Additionally, a FDR adjustment, to avoid type I error in multiple correlations, was done. The $90 \%$ (9 of 10 total) of the correlations were positive (Table 2) and passed the FDR adjustment at 5\% (graphed in Figure 5).

We found that $\mathrm{NO}^{-}{ }_{2}$ levels in plasma were positively correlated with disinhibition measures (Figure 5.A).

The analyses also indicate a possible relationship between the cortical alterations and the disability in behavioral inhibition found. Specifically, elevations in frontal cortex key parameters of lipid peroxidation (4-HNE), apoptosis (caspase 9) and cell damage (HSP70, HMGB1) positively correlated with disinhibited-like behavior observed in the EPM (Figure 5.B.C.D).

Table 2. Correlational, linear regression and FDR analyses between key damage markers and behavioral parameters in the CA+TDD model. [Pearson's coefficient correlation $r$; Slope and constant from the linear regression analyses, $p$-value for both correlation and linear regression and q-value from FDR analyses]. Corrected p-values by using the Benjamini-Hochberg procedure (FDR set at 0.05 ) to restrict the type I error in multiple comparisons. Significant correlations ( $\left.{ }^{*} p<0.05\right)$ and FDR-corrected $p$ values $(\# q<0.05)$.

\begin{tabular}{cccccc|ccccc}
\hline \multicolumn{10}{c}{ Percentages in open arms in the Elevated Plus Maze } \\
\hline $\begin{array}{c}\text { Protein } \\
\text { marker }\end{array}$ & $r$ & Slope & Constant & $p$ value & qvalue & $r$ & Slope & Constant & $p$ value & q value \\
\hline $\begin{array}{c}\text { Plasma } \\
\text { NO- }^{-}\end{array}$ & 0.5044 & 0.0005792 & -0.00688 & 0.0659 & 0.0659 & 0.6692 & 0.0008195 & -0.03051 & 0.0089 & 0.0111 \\
\hline 4-HNE & 0.7596 & 0.001149 & -0.09649 & 0.001 & 0.002 & 0.7128 & 0.001158 & -0.07876 & 0.0029 & 0.0043 \\
Caspase-9 & 0.7611 & 0.002541 & -0.2306 & 0.001 & 0.002 & 0.7633 & 0.002739 & -0.2364 & 0.0009 & 0.002 \\
HSP70 & 0.7952 & 0.0118 & -1.169 & 0.0004 & 0.002 & 0.8949 & 0.01427 & -1.413 & $<0.0001$ & 0.001 \\
HMGB1 & 0.5783 & 0.01111 & -1.07 & 0.0384 & 0.0427 & 0.752 & 0.01704 & -1.659 & 0.003 & 0.0043 \\
\hline
\end{tabular}

${ }^{1}$ Ratios from EPM: Open time/total time; Open arms entries/total entries.

${ }^{2,+}$ All protein markers were detected in frontal cortex, except for nitrites, which were determined in plasma. 


\section{(A) Disinhibition - nitrosative stress in plasma}

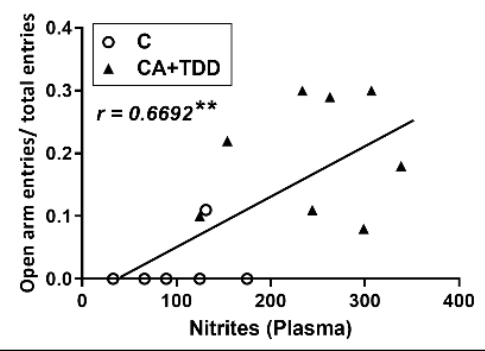

(B) Disinhibition - cortical lipid peroxidation
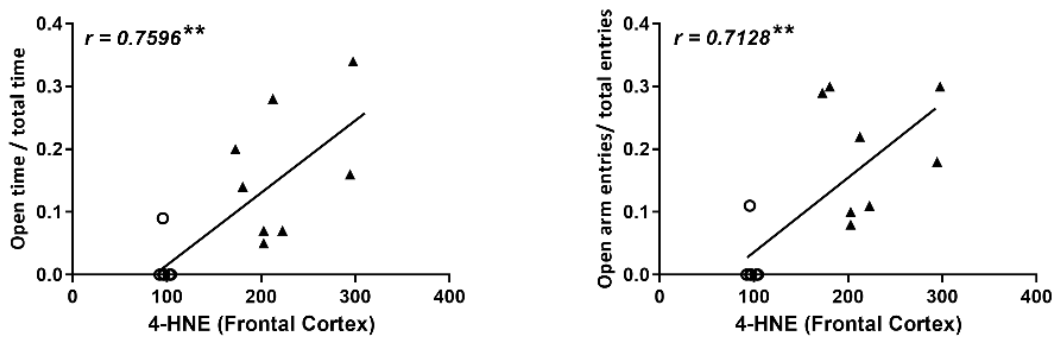

(C)

Disinhibition - cortical apoptosis
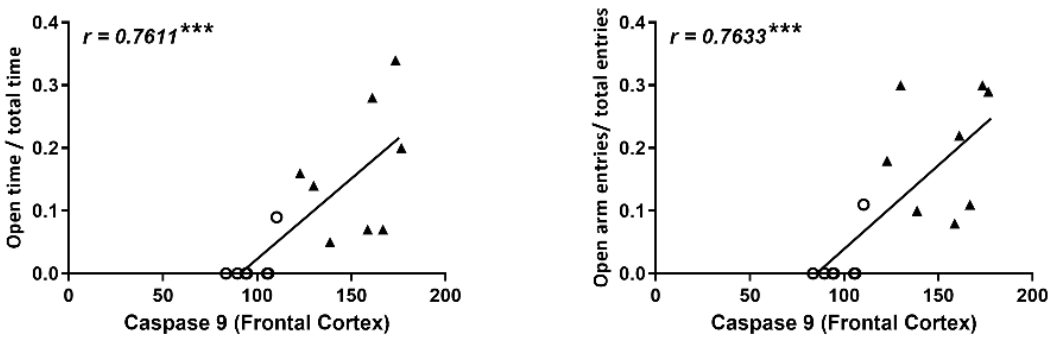

(D) Disinhibition - cortical cell damage
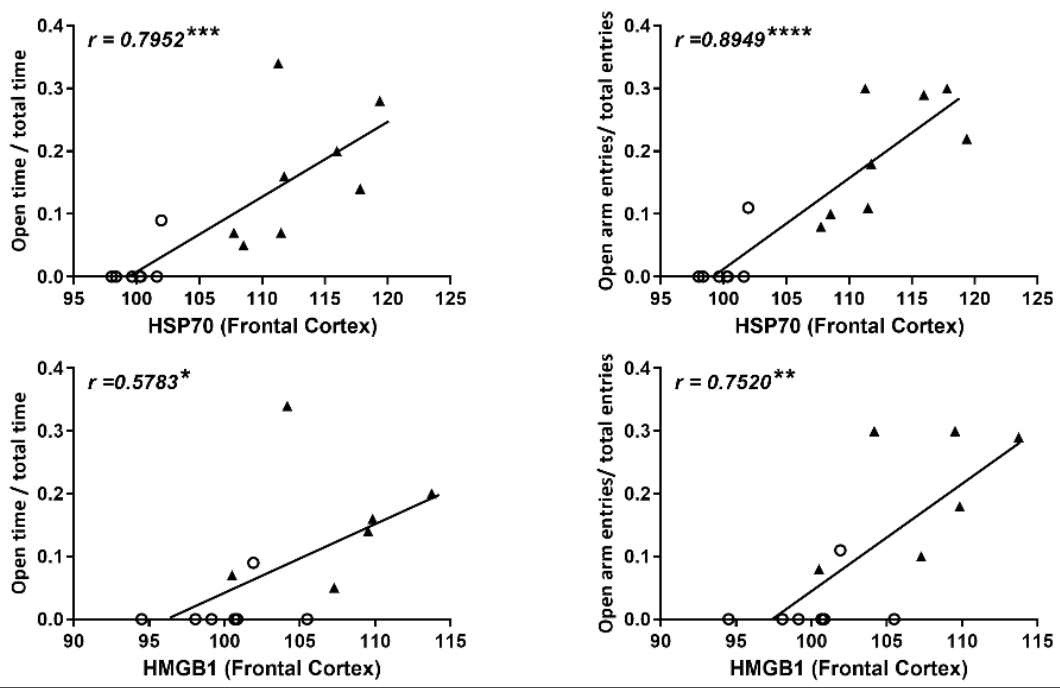

Figure 5. Correlations between damage parameters and disinhibition measures of the EPM in the CA+TDD-treated animals. The trend lines on each graph show the combined regression analyses for the Control (circles) and CA+TDD (triangles) groups. (A) $\mathrm{NO}^{-}{ }_{2}$ levels in plasma were positively correlated with the EPM Open entries ratio. Frontal cortical levels of (B) 4-HNE, (C) Caspase 9 and (D) HSP70, HMGB1 were positively correlated with the EPM Open time and entries ratios. Pearson's coefficient correlation $r$; ${ }^{*} \mathrm{p}<$ $0.05,{ }^{* *} \mathrm{p}<0.01,{ }^{* * *} \mathrm{p}<0.001,{ }^{* * * *} \mathrm{p}<0.0001$. 


\section{Discussion}

In this study WKS was modeled by CA consumption, since the AUD is the main cause of this pathology, with and without TD diet, to understand the contribution of alcohol and the nutritional deficiency of thiamine to the pathology. Our results showed that CA influenced locomotion and memory, but only in conjunction with TDD caused pronounced disinhibited-like behavior. Likewise, the combination of both treatments led to the greatest alterations in the peripheral and cortical markers of oxidative/nitrosative stress and cellular/apoptotic damage. Such injury signals correlated with the inhibitory deficit, suggesting that the frontal cortex is a vulnerable brain area targeting the combined effects of CA and TDD on cell viability and behavior.

Early ethanol exposure leads to a greater propensity for the development of AUDs [37]. In the present work, the age of the animals appeared to influence the observed pattern of alcohol consumption. Adolescent onset (around PD 40) of chronic drinking led to higher alcohol intake compared to later stages. This is consistent with previous studies of alcohol exposure rats where adolescents drank more and displayed an increased metabolic rate, compared to adult rats [23].

In relation to neurological signs, the characteristic symptomatology induced by TD has been extensively demonstrated in rodents, displaying ataxia (i.e., gait problems), loss of righting reflex, and the emergence of seizures and opisthotonus [38-40]. In our previous study with TD-induced WKS models [18], we observed clear motor impairments related with cerebellar dysfunction between 14 and 16 days of TD, but at 12 days the animals are still in a presymptomatic state. In accordance with this work, the 12 days of TD diet appeared not to be enough to cause severe neurological signs, even in combination with CA treatment. This could be associated with the absence of alterations in the cerebellum of these animals (unpublished results). Although there is previous evidence pointing to the cerebellum as a vulnerable structure to alcohol and TD [41,42], here we did not find TLR4neuroinflammatory signature in any of the treatment conditions. Future studies will be carried out in this regard.

Nevertheless, we consider interesting to point out the lower capacity to react to the startle reflex found in some CA animals compared to controls. In line with our results, previous studies reported that ethanol affects markedly the startle response in rats by a mechanism involving central dopaminergic and serotoninergic neurons [43].

In addition, we observed a marked increase in the locomotor activity (SAT, OFT) as a consequence of the CA treatment. These findings could be explained by the development of sensitization to the locomotor stimulant effects of alcohol. This could reflect an increase in alcohol "wanting", thus playing an important role in addiction [44]. Certainly, this phenomenon has been more studied in mice, versus just a few works reported in rats [45-47]. Thereby, our study also extends these previous reports in rats.

Alcohol-related neuropsychological impairments have been widely described in the literature, being executive functions, working and episodic memory frequently affected [48]. Many experimental paradigms have been used to evaluate memory deficits in alcohol-treated rats. However, several of these works show that depending on the treatment protocol, long-term alcohol intake does not necessarily lead to cognitive impairment [49]. In our research, a chronic pattern of alcohol consumption did not appear to affect the spatial memory of the animals (SAT). Nevertheless, controversial results have been reported following CA treatment, since Vedder and colleagues, 2015 [50], also using the spontaneous alternation task, found deficits in spatial memory of their animals. It is possible that the discrepancies in the results obtained under similar experimental conditions might depend on differences in the duration of alcohol treatment, BECs achieved, the presence or absence of a withdrawal period following ethanol administration, the age and strain of the rats, etc.

Regarding another kind of memory task analyzed, CA animals exhibited a deficit in recognition memory (NOT), in line with previous research showing that alcohol intake impairs the performance of rats on the object recognition test $[49,51]$. 
Thus, our results appear to indicate that CA interferes with recognition memory but not with spatial memory. A possible interpretation of these findings could be that the mentioned tasks recruit different brain structures. Spatial memory performance is more dependent on the hippocampal function than does the recognition memory [52]. By contrast, recognition memory appears to be more dependent on cortical structures, as medial prefrontal cortex [53]. According to this, it has been demonstrated that the prefrontal cortex is more vulnerable to chronic alcohol-induced toxicity than the hippocampus [54]. Thus, recognition memory would be more sensitive to the cortical damage found in this study, whereas the hippocampus could be less affected. Further studies are needed to clarify this point.

In addition, TDD alone did not affect any type of memory studied, and there was not any additive or synergistic interaction between CA+TDD in memory performance either. In contrast, we found that exclusively CA combined with TDD induced a pronounced disinhibited-like behavior (EPM). CA+TDD rats exhibited an increase in the percentage of entries and of time spent in the open arms, relative to controls, interpreted as increased risk-taking behaviour and/or impulsivity [32,33]. This effect is not observed by CA exposure alone, so this may indicate that TDD is necessary to develop an impulsive o disinhibited behavior. In fact, we recently reported such disinhibitory behavior in a non-alcoholic WKS model based in TD diet, where TD-induced brain damage was exacerbated by glucose loading [18]. Furthermore, we also found this disinhibitory effect in the OFT, since the CA+TDD animals appeared to show a lack of environmental awareness by walking and spending more time in the arena center.

Disinhibition reflect a deficit in executive functioning, which is associated with frontal brain damage [10]. This has been revealed as one of the most important alterations suffered by WKS patients, with devastating emotional and social consequences [9]. Moreover, impulse control deficit is associated with addiction [55].

The frontal cortex is responsible for executive functions and inhibition of impulsive responses and is particularly sensitive to alcohol and TD-induced damage $[\mathbf{1 6}, \mathbf{1 8 , 5 4 , 5 6 ]}$. In particular, it has been shown that alcohol and TD lead to the upregulated expression of the iNOS enzyme $[\mathbf{1 7}, \mathbf{1 8}, \mathbf{5 7}]$. iNOS can produce large amounts of NO that will react forming reactive nitrogen species (RNS), which are responsible for oxidative/nitrosative stress [58]. These molecules carry out cytotoxic process of lipid peroxidation, releasing toxic products from the degradation of membrane phospholipids such as 4-HNE, leading to cell death.

At the peripheral level, results revealed that CA and TDD individually increased the $\mathrm{NO}^{-}{ }_{2}$ levels in plasma, exerting the combination of both treatments an additive effect in the $\mathrm{NO}^{-}{ }_{2}$ elevation. Due to the location of RNS production from different tissues, plasma nitrites can be a useful global indicator of the degree of damage. However, we found no significant changes in the iNOS levels in the frontal cortex, which can be attributed to the specific timepoint of samples collection. Nevertheless, CA exposure did increase the 4HNE levels in the frontal cortex, observing, again, a greater increase with the combined treatment (CA with TDD).

Consequently, ethanol-induced oxidative stress can be responsible for apoptosis and neuronal dysfunction (reviewed in [1,54]. In contrast, several studies showed that TD triggers more oxidative stress and severe cellular death than alcohol. In turn, ethanol and TD showed additive/synergistic toxicity on cellular death [59]. Recent studies indicate that TD can use caspase-3 classical pathway to amplify apoptosis as alcohol intoxication, although both alcohol and TD can induce cellular death by different pathways (reviewed in [60]. Taking into account our data, these observations may depend on the pattern of alcohol consumption and the degree of TD. We found upregulation of caspases 3 and 8 in our previous studies with alcohol binge drinking, as precursors of the emergence of damage molecules such as HMGB1 [17]. Here, CA+TDD treatment did not modify the levels of 
caspase 3 and 8 in the frontal cortex, although a trend towards upregulation can be observed. Again, the time-course of expression of such parameters may influence these results. However, caspase 9 appeared to be more susceptible to upregulation by all the treatments, showing no additive effect by the combined CA+TDD treatment, which can be due to a ceiling effect in the caspase expression.

In addition, alcohol and TD have been involved in the release of damage-associated molecular patterns (DAMPs) from cells in response to cellular stress or tissue injury $[17,18]$. DAMPs as HSP60-70 and HMGB-1 active the innate immune system through TLR2 and TLR4 signaling. Thus, these molecules can lead to a vicious cycle by amplifying the neuroinflammation and inducing more cell damage (reviewed in [1]). Our findings showed that the combination of CA with TDD induced an increase in the HSP70 and HMGB1 cortical levels. The TDD main effect upregulating HMGB1 may suggest a greater influence of this treatment.

In view of our results, we can conclude that CA in conjunction with TDD led to the most severe cortical, peripheral and behavioral alterations. In accordance, higher levels of cortical lipid peroxidation, apoptotic and cell damage markers, as well as peripheral $\mathrm{NO}^{-}$, correlated with the open arms exploration in the EPM, suggesting a link between the cortical injury and the disinhibited-like behavior, a core symptom of the pathology, as discussed above.

\section{Conclusions}

Our study models WKS through CA consumption, TD or the combination of both. The animal model based in CA consumption showed a sensitization to the hyperlocomotor effect of alcohol together with a recognition memory deficit, but only in conjunction with TDD the disinhibited-like behaviour, a core characteristic of the pathology, is induced. CA and TDD individually caused selective cortical damage and an increase of $\mathrm{NO}^{-} 2$ in plasma, whereas the combination of these treatments aggravated the alterations in the markers of nitrosative stress, lipid peroxidation, apoptosis and cell damage studied. Thus, these processes appear to play a critical role in the cortical injury that could be related to the deficit in its inhibitory function. This way, the nutritional deficiency appears to be implicated in the production of the disinhibited behavior, which is even more limiting in the daily life of WKS patients than memory problems.

Therefore, our study shed light on the underlying disease-specific mechanisms, reinforcing the need for neuroprotective therapeutic approaches with antioxidant and antiapoptotic actions, along with preventive treatments of the nutritional deficiency in WKS.

Author Contributions: Conceptualization: MM, GBG and LO.; methodology and investigation: MM and LLV; formal analysis and data curation: MM, BGB and LO; writing - original draft preparation: MM; writing - review and editing and funding acquisition: LO. All authors have read and agreed to the published version of the manuscript."

Funding: This work was supported by FEDER (European Union)/ MINISTERIO DE CIENCIA E INNOVACIÓN (MICINN) / Agencia Estatal de Investigación (Spain), grant number: Retos 2018; RTI2018-099535-B-I00 to L.O.

Institutional Review Board Statement: The study was conducted according to the guidelines of the Declaration of Helsinki, and approved by the Animal Welfare Committee of the Complutense University of Madrid (reference: PROEX $312 / 19,26^{\text {th }}$ April, 2020) and according to European legislation (2010/63/UE).

Data Availability Statement: The data presented in this study are available on request from the corresponding author.

Conflicts of Interest: The authors declare no conflict of interest. 


\section{Appendix A}

\section{Supplemental discussion of the body weight changes found}

In the present work with young growing rats, we observed that the body weight of CA rats was always significantly lower than in controls. In this regard, prolonged alcohol consumption is related to an increased risk of nutritional deficiency or malnutrition. Alcohol effects on the stomach and intestines may decrease absorption and utilization of nutrients, while increasing its excretion. In accordance with our findings, it has been shown that prolonged alcohol intake may result in lower body mass gain in animal experiments in comparison with animals that do not receive alcohol (reviewed in [61]). The reduced body weight gain observed in young rats drinking alcohol could be due to loss of appetite with lower dietary intake or increased energy expenditures [62]. Simultaneously, in the case of growing organisms, the influence of alcohol consumption on growth hormone level may be especially important and may be decreased by ethanol consumption, with a resultant reduction in bodily growth (reviewed in [61]. In addition, its known that TD reduces food consumption and body weight in rodents $[18,63]$, which is in accordance with our results. Regarding to this, it has been proposed that thiamine, as well as alcohol, could play a main role regulating the increase in energy expenditure [63].

\section{Appendix B}

Thiamine: plasma levels and oral supplementation treatment. Results and conclusions

Analysis of levels of thiamine in plasma from initial (1 month) and intermediate (3 months) time-points showed no significant differences neither between nor within controls and CA groups:

week 4 : control $=55.5 \pm 6.28 \mathrm{ng} / \mathrm{mL} ; \mathrm{CA}=55.82 \pm 7.31 \mathrm{ng} / \mathrm{mL}$;

week 12: control $=49.37 \pm 4.8 \mathrm{ng} / \mathrm{mL} ; \mathrm{CA}=52.06 \pm 9.55 \mathrm{ng} / \mathrm{mL}$;

(two-way RM ANOVA, interaction: $\mathrm{F}(1,20)=0.03686$; effect of time: $\mathrm{F}(1,20)=0.6462$; effect of treatment: $\mathrm{F}(1,20)=0.03426 ; \mathrm{p}>0.05$, n.s).

However, in the final measurement, after a long period (9 months) of exposure to alcohol and after TD diet treatment, we found a trend towards a decrease in total thiamine levels due to an alcohol effect. In Figure 6, the thiamine increment [ $\Delta$ Thiamine] was calculated for each group between the two time-points (final and intermediate levels) during the adult stage (because of similar metabolism). Due to significant weight loss in TDD and CA+TDD animals, values of thiamine levels were weight-corrected (ng/mL per kilogram) (two-way ANOVA, main effect of alcohol $\mathrm{F}(1,24)=3.411, \mathrm{p}=0.0771$ ).

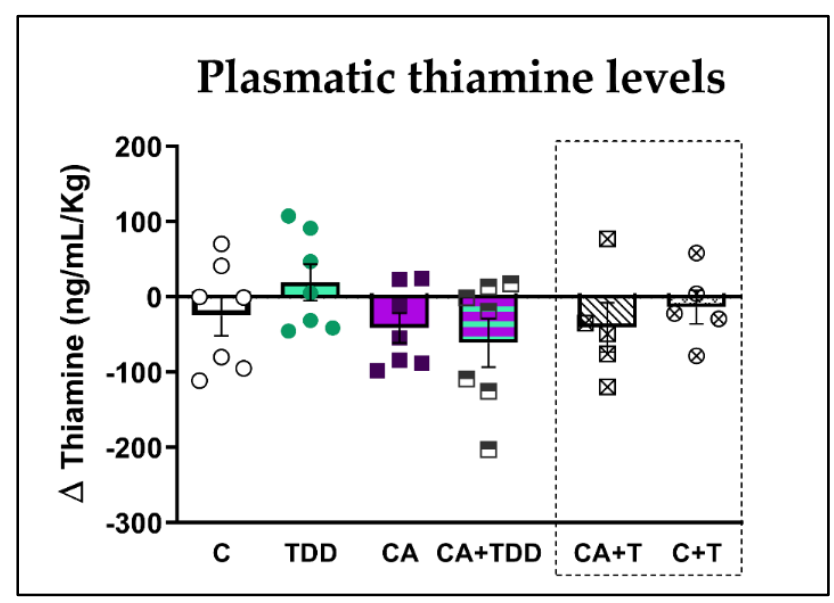

Figure 6. Decrement in plasma levels of total thiamine at the end of the protocol (36 weeks) versus intermediate status (week 12) in adult rats. Results normalized by body weight $[\mathrm{ng} / \mathrm{mL}$ data divided by kilogram]. Data are expressed as mean \pm S.E.M. 
As it has been previously mentioned, chronic alcohol consumption lead to nutritional deficiency as TD. Our results showed a trend towards a decline in the thiamine levels in plasma in CA rats. Nevertheless, we analyzed total levels of thiamine and not its active form (thiamine diphosphate, TDP) as it has been studied in other works [50,56]. Zahr and colleagues, 2014 [64] reported that total thiamine concentrations were not sensitive to thiamine deprivation, but they found significant lower levels of its metabolites TDP. Accordingly, it would be expected that the active forms of this vitamin would be more affected by the treatments.

In the present experiment, in addition to the 4 experimental groups reported in the result section, we also included a group receiving CA with oral thiamine supplementation to deduce whether the effects of long term alcohol exposure can be attenuated with prophylactic treatment. This supplementing thiamine apparently did not restore total thiamine levels in CA+T-treated rats (Figure 6), and these animals did not show improvement in any of the behavioral and biochemical variables analyzed with respect to the CA group either.

Thus, this indicate that the oral treatment at this dose of thiamine did not prevent the effects of CA that could be caused by alcohol-induced TD itself. Although there are no universally accepted guidelines, recommended treatment of WKS consists of thiamine administration, preferably by intravenous route, followed by oral administration [65]. As described above in the introduction of this article, CA exposure can impair the gastric absorption and cellular utilization of thiamine. However, the oral route is suitable for repeated and prolonged use, as it is the simplest, most convenient and pain-free.

In addition to thiamine supplementation other oral treatment alternatives have been proposed. For example, the benfotiamine is a pro-vitamin B1 with greater absorption and bioavailability compared to orally administered thiamine, producing higher thiamine (and also thiamine mono and diphosphate) concentration after alcohol exposure [66]. 


\section{References}

1. Orio, L.; Alen, F.; Pavón, F.J.; Serrano, A.; García-Bueno, B. Oleoylethanolamide, Neuroinflammation, and Alcohol Abuse. Front. Mol. Neurosci. 2019, 11, 490, doi:10.3389/fnmol.2018.00490.

2. Arts, N.J.; Walvoort, S.J.; Kessels, R.P. Korsakoff's Syndrome: A Critical Review. Neuropsychiatr Dis Treat 2017, 13, 2875-2890, doi:10.2147/NDT.S130078.

3. Zahr, N.M.; Kaufman, K.L.; Harper, C.G. Clinical and Pathological Features of Alcohol-Related Brain Damage. Nat Rev Neurol 2011, 7, 284-294, doi:10.1038/nrneurol.2011.42.

4. Kopelman, M.D. The Korsakoff Syndrome. Br J Psychiatry 1995, 166, 154-173, doi:10.1192/bjp.166.2.154.

5. Deb, S.; Law-Min, R.; Fearnley, D. Wernicke-Korsakoff Syndrome Following Small Bowel Obstruction. Behav Neurol 2001, 13, 89-94, doi:10.1155/2002/702526.

6. Jhala, S.S.; Hazell, A.S. Modeling Neurodegenerative Disease Pathophysiology in Thiamine Deficiency: Consequences of Impaired Oxidative Metabolism. Neurochem Int 2011, 58, 248-260, doi:10.1016/j.neuint.2010.11.019.

7. Jones, D.C.; Houts, R. Parental Drinking, Parent-Child Communication, and Social Skills in Young Adults. J Stud Alcohol 1992, 53, 48-56, doi:10.15288/jsa.1992.53.48.

8. Morgenstern, J.; Bates, M.E. Effects of Executive Function Impairment on Change Processes and Substance Use Outcomes in 12-Step Treatment. J Stud Alcohol 1999, 60, 846-855, doi:10.15288/jsa.1999.60.846.

9. Gerridzen, I.J.; Hertogh, C.M.P.M.; Depla, M.F.; Veenhuizen, R.B.; Verschuur, E.M.L.; Joling, K.J. Neuropsychiatric Symptoms in People With Korsakoff Syndrome and Other Alcohol-Related Cognitive Disorders Living in Specialized Long-Term Care Facilities: Prevalence, Severity, and Associated Caregiver Distress. J Am Med Dir Assoc 2018, 19, 240-247, doi:10.1016/j.jamda.2017.09.013.

10. Knutson, K.M.; Dal Monte, O.; Schintu, S.; Wassermann, E.M.; Raymont, V.; Grafman, J.; Krueger, F. Areas of Brain Damage Underlying Increased Reports of Behavioral Disinhibition. J Neuropsychiatry Clin Neurosci 2015, 27, 193-198, doi:10.1176/appi.neuropsych.14060126.

11. Jacobson, R.R.; Lishman, W.A. Cortical and Diencephalic Lesions in Korsakoff's Syndrome: A Clinical and CT Scan Study. Psychol Med 1990, 20, 63-75, doi:10.1017/s0033291700013234.

12. Jernigan, T.L.; Schafer, K.; Butters, N.; Cermak, L.S. Magnetic Resonance Imaging of Alcoholic Korsakoff Patients. Neuropsychopharmacology 1991, 4, 175-186.

13. Paller, K.A.; Acharya, A.; Richardson, B.C.; Plaisant, O.; Shimamura, A.P.; Reed, B.R.; Jagust, W.J. Functional Neuroimaging of Cortical Dysfunction in Alcoholic Korsakoff's Syndrome. J Cogn Neurosci 1997, 9, 277-293, doi:10.1162/jocn.1997.9.2.277.

14. Aupée, A.M.; Desgranges, B.; Eustache, F.; Lalevée, C.; de la Sayette, V.; Viader, F.; Baron, J.C. Voxel-Based Mapping of Brain Hypometabolism in Permanent Amnesia with PET. Neuroimage 2001, 13, 1164-1173, doi:10.1006/nimg.2001.0762.

15. Knight RG; Longmore BE The Wernicke-Korsakoff Syndrome. Clinical neuropsychology of alcoholism $267-311$.

16. Nunes, P.T.; Kipp, B.T.; Reitz, N.L.; Savage, L.M. Aging with Alcohol-Related Brain Damage: Critical Brain Circuits Associated with Cognitive Dysfunction. Int. Rev. Neurobiol. 2019, 148, 101-168, doi:10.1016/bs.irn.2019.09.002.

17. Antón, M.; Alén, F.; Gómez de Heras, R.; Serrano, A.; Pavón, F.J.; Leza, J.C.; García-Bueno, B.; Rodríguez de Fonseca, F.; Orio, L. Oleoylethanolamide Prevents Neuroimmune HMGB1/TLR4/NF-KB Danger Signaling in Rat Frontal Cortex and Depressive-like Behavior Induced by Ethanol Binge Administration: OEA Blocks Ethanol TLR4 Signaling. Addiction Biology 2017, 22, 724-741, doi:10.1111/adb.12365.

18. Moya, M.; San Felipe, D.; Ballesta, A.; Alén, F.; Rodríguez de Fonseca, F.; García-Bueno, B.; Marco, E.M.; Orio, L. Cerebellar and Cortical TLR4 Activation and Behavioral Impairments in Wernicke-Korsakoff Syndrome: 
Pharmacological Effects of Oleoylethanolamide. Prog Neuropsychopharmacol Biol Psychiatry 2021, 108, 110190, doi:10.1016/j.pnpbp.2020.110190.

19. Fadda, F.; Rossetti, Z.L. Chronic Ethanol Consumption: From Neuroadaptation to Neurodegeneration. Prog Neurobiol 1998, 56, 385-431, doi:10.1016/s0301-0082(98)00032-x.

20. Harper, C.; Matsumoto, I. Ethanol and Brain Damage. Curr Opin Pharmacol 2005, 5, 73-78, doi:10.1016/j.coph.2004.06.011.

21. Pfefferbaum, A.; Sullivan, E.V.; Mathalon, D.H.; Lim, K.O. Frontal Lobe Volume Loss Observed with Magnetic Resonance Imaging in Older Chronic Alcoholics. Alcohol Clin Exp Res 1997, 21, 521-529, doi:10.1111/j.15300277.1997.tb03798.x.

22. Tsao, W.-C.; Ro, L.-S.; Chen, C.-M.; Chang, H.-S.; Kuo, H.-C. Non-Alcoholic Wernicke's Encephalopathy with Cortical Involvement and Polyneuropathy Following Gastrectomy. Metab Brain Dis 2017, 32, 1649-1657, doi:10.1007/s11011-017-0055-8.

23. Fernandez, G.M.; Stewart, W.N.; Savage, L.M. Chronic Drinking During Adolescence Predisposes the Adult Rat for Continued Heavy Drinking: Neurotrophin and Behavioral Adaptation after Long-Term, Continuous Ethanol Exposure. PLoS One 2016, 11, e0149987, doi:10.1371/journal.pone.0149987.

24. Bâ, A.; Seri, B.V.; Han, S.H. Thiamine Administration during Chronic Alcohol Intake in Pregnant and Lactating Rats: Effects on the Offspring Neurobehavioural Development. Alcohol Alcohol 1996, 31, 27-40, doi:10.1093/oxfordjournals.alcalc.a008113.

25. Bâ, A.; Seri, B.V.; Aka, K.J.; Glin, L.; Tako, A. Comparative Effects of Developmental Thiamine Deficiencies and Ethanol Exposure on the Morphometry of the CA3 Pyramidal Cells. Neurotoxicol Teratol 1999, 21, 579-586, doi:10.1016/s0892-0362(99)00014-8.

26. Bâ, A. Alcohol and B1 Vitamin Deficiency-Related Stillbirths. J Matern Fetal Neonatal Med 2009, 22, 452-457, doi:10.1080/14767050802609775.

27. Bâ, A. Comparative Effects of Alcohol and Thiamine Deficiency on the Developing Central Nervous System. Behav Brain Res 2011, 225, 235-242, doi:10.1016/j.bbr.2011.07.015.

28. Bâ, A. Paradoxical Effects of Alcohol and Thiamine Deficiency on the Eye Opening in Rat Pups. J Matern Fetal Neonatal Med 2012, 25, 2435-2440, doi:10.3109/14767058.2012.703712.

29. Jantzie, L.L.; Oppong, A.Y.; Conteh, F.S.; Yellowhair, T.R.; Kim, J.; Fink, G.; Wolin, A.R.; Northington, F.J.; Robinson, S. Repetitive Neonatal Erythropoietin and Melatonin Combinatorial Treatment Provides Sustained Repair of Functional Deficits in a Rat Model of Cerebral Palsy. Front Neurol 2018, 9, 233, doi:10.3389/fneur.2018.00233.

30. Bouwknecht, J.A.; Spiga, F.; Staub, D.R.; Hale, M.W.; Shekhar, A.; Lowry, C.A. Differential Effects of Exposure to Low-Light or High-Light Open-Field on Anxiety-Related Behaviors; Relationship to c-Fos Expression in Serotonergic and Non-Serotonergic Neurons in the Dorsal Raphe Nucleus. Brain Res Bull 2007, 72, 32-43, doi:10.1016/j.brainresbull.2006.12.009.

31. Cosquer, B.; Galani, R.; Kuster, N.; Cassel, J.-C. Whole-Body Exposure to 2.45 GHz Electromagnetic Fields Does Not Alter Anxiety Responses in Rats: A plus-Maze Study Including Test Validation. Behav Brain Res 2005, 156, 6574, doi:10.1016/j.bbr.2004.05.007.

32. Davis, J.F.; Krause, E.G.; Melhorn, S.J.; Sakai, R.R.; Benoit, S.C. Dominant Rats Are Natural Risk Takers and Display Increased Motivation for Food Reward. Neuroscience 2009, 162, 23-30, doi:10.1016/j.neuroscience.2009.04.039.

33. Joshi, U.; Evans, J.E.; Joseph, R.; Emmerich, T.; Saltiel, N.; Lungmus, C.; Oberlin, S.; Langlois, H.; Ojo, J.; Mouzon, B.; et al. Oleoylethanolamide Treatment Reduces Neurobehavioral Deficits and Brain Pathology in a Mouse Model of Gulf War Illness. Sci Rep 2018, 8, 12921, doi:10.1038/s41598-018-31242-7. 
34. Dember, W.N.; Fowler, H. Spontaneous Alternation Behavior. Psychol Bull 1958, 55, 412-428, doi:10.1037/h0045446.

35. Marco, E.M.; Valero, M.; de la Serna, O.; Aisa, B.; Borcel, E.; Ramirez, M.J.; Viveros, M.-P. Maternal Deprivation Effects on Brain Plasticity and Recognition Memory in Adolescent Male and Female Rats. Neuropharmacology 2013, 68, 223-231, doi:10.1016/j.neuropharm.2012.08.014.

36. Green, L.C.; Wagner, D.A.; Glogowski, J.; Skipper, P.L.; Wishnok, J.S.; Tannenbaum, S.R. Analysis of Nitrate, Nitrite, and [15N]Nitrate in Biological Fluids. Anal Biochem 1982, 126, 131-138, doi:10.1016/0003-2697(82)90118-x.

37. DeWit, D.J.; Adlaf, E.M.; Offord, D.R.; Ogborne, A.C. Age at First Alcohol Use: A Risk Factor for the Development of Alcohol Disorders. Am J Psychiatry 2000, 157, 745-750, doi:10.1176/appi.ajp.157.5.745.

38. Anzalone, S.; Vetreno, R.P.; Ramos, R.L.; Savage, L.M. Cortical Cholinergic Abnormalities Contribute to the Amnesic State Induced by Pyrithiamine-Induced Thiamine Deficiency in the Rat: Cortical Cholinergic Abnormalities. European Journal of Neuroscience 2010, 32, 847-858, doi:10.1111/j.1460-9568.2010.07358.x.

39. Gu, B.; Desjardins, P.; Butterworth, R.F. Selective Increase of Neuronal Cyclooxygenase-2 (COX-2) Expression in Vulnerable Brain Regions of Rats with Experimental Wernicke's Encephalopathy: Effect of Nimesulide. Metab Brain Dis 2008, 23, 175-187, doi:10.1007/s11011-008-9089-2.

40. Vetreno, R.P.; Ramos, R.L.; Anzalone, S.; Savage, L.M. Brain and Behavioral Pathology in an Animal Model of Wernicke's Encephalopathy and Wernicke-Korsakoff Syndrome. Brain Research 2012, 1436, 178-192, doi:10.1016/j.brainres.2011.11.038.

41. Harper, C. The Neuropathology of Alcohol-Related Brain Damage. Alcohol Alcohol 2009, 44, 136-140, doi:10.1093/alcalc/agn102.

42. Sullivan, E.V.; Pfefferbaum, A. Neurocircuitry in Alcoholism: A Substrate of Disruption and Repair. Psychopharmacology (Berl) 2005, 180, 583-594, doi:10.1007/s00213-005-2267-6.

43. Pohorecky, L.A.; Cagan, M.; Brick, J.; Jaffe, S.L. The Startle Response in Rats: Effect of Ethanol. Pharmacol Biochem Behav 1976, 4, 311-316, doi:10.1016/0091-3057(76)90247-1.

44. Legastelois, R. Sensitization to the Stimulant Motor Effects of Ethanol Is Not Dependent On Tolerance to Ataxic or Sedative Properties of Ethanol in Female Mice. J Alcohol Drug Depend 2015, 03, doi:10.4172/2329-6488.1000216.

45. Goldstein KR; Knapp DJ; Saiff EI; Pohorecky L; Benjamin D Sensitization to Ethanol Demonstrated in PlacePreference and Locomotor Activation. Soc Neurosci Abstr 18:107.

46. Hoshaw, B.A.; Lewis, M.J. Behavioral Sensitization to Ethanol in Rats: Evidence from the Sprague-Dawley Strain. Pharmacol Biochem Behav 2001, 68, 685-690, doi:10.1016/s0091-3057(01)00489-0.

47. Xu, S.; Kang, U.G. Characteristics of Ethanol-Induced Behavioral Sensitization in Rats: Molecular Mediators and Cross-Sensitization between Ethanol and Cocaine. Pharmacol Biochem Behav 2017, 160, 47-54, doi:10.1016/j.pbb.2017.08.001.

48. Maillard, A.; Cabé, N.; Viader, F.; Pitel, A.-L. Neuropsychological Deficits in Alcohol Use Disorder. In; 2020; pp. 103-128 ISBN 978-0-12-815298-0.

49. García-Moreno, L.M.; Conejo, N.M.; Capilla, A.; García-Sánchez, O.; Senderek, K.; Arias, J.L. Chronic Ethanol Intake and Object Recognition in Young and Adult Rats. Prog Neuropsychopharmacol Biol Psychiatry 2002, 26, 831837, doi:10.1016/s0278-5846(01)00327-x.

50. Vedder, L.C.; Hall, J.M.; Jabrouin, K.R.; Savage, L.M. Interactions between Chronic Ethanol Consumption and Thiamine Deficiency on Neural Plasticity, Spatial Memory, and Cognitive Flexibility. Alcohol Clin Exp Res 2015, 39, 2143-2153, doi:10.1111/acer.12859.

51. Ciccocioppo, R.; Antonelli, L.; Biondini, M.; Perfumi, M.; Pompei, P.; Massi, M. Memory Impairment Following Combined Exposure to Delta(9)-Tetrahydrocannabinol and Ethanol in Rats. Eur J Pharmacol 2002, 449, 245-252, doi:10.1016/s0014-2999(02)01999-4. 
52. Broadbent, N.J.; Squire, L.R.; Clark, R.E. Spatial Memory, Recognition Memory, and the Hippocampus. Proc Natl Acad Sci U S A 2004, 101, 14515-14520, doi:10.1073/pnas.0406344101.

53. Morici, J.F.; Bekinschtein, P.; Weisstaub, N.V. Medial Prefrontal Cortex Role in Recognition Memory in Rodents. Behav Brain Res 2015, 292, 241-251, doi:10.1016/j.bbr.2015.06.030.

54. Fowler, A.-K.; Thompson, J.; Chen, L.; Dagda, M.; Dertien, J.; Dossou, K.S.S.; Moaddel, R.; Bergeson, S.E.; Kruman, I.I. Differential Sensitivity of Prefrontal Cortex and Hippocampus to Alcohol-Induced Toxicity. PLoS One 2014, 9, e106945, doi:10.1371/journal.pone.0106945.

55. Crews, F.T.; Boettiger, C.A. Impulsivity, Frontal Lobes and Risk for Addiction. Pharmacol Biochem Behav 2009, 93, 237-247, doi:10.1016/j.pbb.2009.04.018.

56. Toledo Nunes, P.; Vedder, L.C.; Deak, T.; Savage, L.M. A Pivotal Role for Thiamine Deficiency in the Expression of Neuroinflammation Markers in Models of Alcohol-Related Brain Damage. Alcohol. Clin. Exp. Res. 2019, 43, 425438, doi:10.1111/acer.13946.

57. Gong, Y.-S.; Hu, K.; Yang, L.-Q.; Guo, J.; Gao, Y.-Q.; Song, F.-L.; Hou, F.-L.; Liang, C.-Y. Comparative Effects of EtOH Consumption and Thiamine Deficiency on Cognitive Impairment, Oxidative Damage, and $\beta$-Amyloid Peptide Overproduction in the Brain. Free Radic Biol Med 2017, 108, 163-173, doi:10.1016/j.freeradbiomed.2017.03.019.

58. Pacher, P.; Beckman, J.S.; Liaudet, L. Nitric Oxide and Peroxynitrite in Health and Disease. Physiol Rev 2007, 87, 315-424, doi:10.1152/physrev.00029.2006.

59. Ke, Z.-J.; Wang, X.; Fan, Z.; Luo, J. Ethanol Promotes Thiamine Deficiency-Induced Neuronal Death: Involvement of Double-Stranded RNA-Activated Protein Kinase. Alcohol Clin Exp Res 2009, 33, 1097-1103, doi:10.1111/j.15300277.2009.00931.x.

60. Bâ, A. Alcohol and Thiamine Deficiency Trigger Differential Mitochondrial Transition Pore Opening Mediating Cellular Death. Apoptosis 2017, 22, 741-752, doi:10.1007/s10495-017-1372-4.

61. Kołota, A.; Głąbska, D.; Oczkowski, M.; Gromadzka-Ostrowska, J. Influence of Alcohol Consumption on Body Mass Gain and Liver Antioxidant Defense in Adolescent Growing Male Rats. Int J Environ Res Public Health 2019, 16, E2320, doi:10.3390/ijerph16132320.

62. Larue-Achagiotis, C.; Poussard, A.M.; Louis-Sylvestre, J. Alcohol Drinking, Food and Fluid Intakes and Body Weight Gain in Rats. Physiol Behav 1990, 47, 545-548, doi:10.1016/0031-9384(90)90124-m.

63. Liu, M.; Alimov, A.P.; Wang, H.; Frank, J.A.; Katz, W.; Xu, M.; Ke, Z.-J.; Luo, J. Thiamine Deficiency Induces Anorexia by Inhibiting Hypothalamic AMPK. Neuroscience 2014, 267, 102-113, doi:10.1016/j.neuroscience.2014.02.033.

64. Zahr, N.M.; Alt, C.; Mayer, D.; Rohlfing, T.; Manning-Bog, A.; Luong, R.; Sullivan, E.V.; Pfefferbaum, A. Associations between in Vivo Neuroimaging and Postmortem Brain Cytokine Markers in a Rodent Model of Wernicke's Encephalopathy. Experimental Neurology 2014, 261, 109-119, doi:10.1016/j.expneurol.2014.06.015.

65. Latt, N.; Dore, G. Thiamine in the Treatment of Wernicke Encephalopathy in Patients with Alcohol Use Disorders. Intern Med J 2014, 44, 911-915, doi:10.1111/imj.12522.

66. Portari, G.V.; Vannucchi, H.; Jordao, A.A. Liver, Plasma and Erythrocyte Levels of Thiamine and Its Phosphate Esters in Rats with Acute Ethanol Intoxication: A Comparison of Thiamine and Benfotiamine Administration. Eur J Pharm Sci 2013, 48, 799-802, doi:10.1016/j.ejps.2013.01.010. 